\title{
Aliased Register Allocation for Straight-line Programs is NP-Complete
}

\author{
Jonathan K. Lee Jens Palsberg Fernando Magno Quintão Pereira \\ UCLA \\ University of California, Los Angeles \\ $\{$ jkenl,palsberg,fernando\}@cs.ucla.edu
}

\begin{abstract}
Register allocation is NP-complete in general but can be solved in linear time for straight-line programs where each variable has at most one definition point if the bank of registers is homogeneous. In this paper we study registers which may alias: an aliased register can be used both independently or in combination with an adjacent register. Such registers are found in commonly-used architectures such as x86, the HP PA-RISC, the Sun SPARC processor, and MIPS floating point. In 2004, Smith, Ramsey, and Holloway presented the best algorithm for aliased register allocation so far; their algorithm is based on a heuristic for coloring of general graphs. Most architectures with register aliasing allow only aligned registers to be combined: for example, the low-address register must have an even number. Open until now is the question of whether working with restricted classes of programs can improve the complexity of aliased register allocation with alignment restrictions. In this paper we show that aliased register allocation with alignment restrictions for straight-line programs is NP-complete. We also present a proof of a related result by Stockmeyer: the shipbuilding problem is NPcomplete
\end{abstract}

\section{Introduction}

Register Allocation. Programmers write most software in high-level programming languages such as $\mathrm{C}, \mathrm{C}++$, and Java, and use compilers to translate their programs to a growing variety of hardware, including multicore platforms, graphics processing units, and network processors. To achieve high execution speed, programmers rely on compilers to optimize the program structure and to use registers and memory in clever ways. The latter task, known as register allocation, has grown steadily in significance because of the widening gap between the short time to access a register and the longer time to access memory. Today, the register allocator may be among the most important and most complicated parts of a compiler. For example, our experiments with the gcc compiler on the StrongARM architecture shows that a good register allocator typically improves execution speed by a factor of 2.5 [14]. A register allocator can also be a significant part of the code of a compiler implementation: $10 \%$ for lcc [9] and $12 \%$ for gcc 2.95.2. 
Most programs use more variables than the number of registers on the target computer. The core of the register allocation problem is to determine whether all the program variables can be placed in machine registers. The reason why a register allocator may be able to place a high number of variables in a small number of registers is that some variables are not live at the same time and so they can share a register. When the need for registers exceeds the number of available registers, the register allocator faces the difficult task of choosing which variables will be placed in registers and which variables will be spilled, that is, placed in memory. In this paper we focus on the core register allocation problem and do not discuss spilling of variables.

Chaitin et al. [4] showed that the core register allocation problem is NPcomplete by a reduction from the graph coloring problem. The essence of Chaitin et al.'s proof is that every graph is the interference graph of some program. Chaitin et al.'s proof assumes a homogeneous bank of registers, where each register can be used to hold the value of any program variable.

\begin{tabular}{|c|c|c|c|c|}
\hline 32 bits & $\overline{\mathrm{AX}}$ & EBX & $\mathrm{ECX}$ & EDX \\
\hline 16 bits & $\mathrm{AX}$ & $\mathrm{BX}$ & $\mathrm{CX}$ & DX \\
\hline 8 bits & \begin{tabular}{l|l}
$\mathrm{AH}$ & $\mathrm{AL}$ \\
\end{tabular} & \begin{tabular}{l|l|}
$\mathrm{BH}$ & $\mathrm{BL}$ \\
\end{tabular} & \begin{tabular}{l|l}
$\mathrm{CH}$ & $\mathrm{CL}$ \\
\end{tabular} & $\mathrm{DH}$ \\
\hline
\end{tabular}

Fig. 1. General purpose registers from the x 86 architecture

Aliased Registers. In this paper we study register allocation for hardware in which the bank of registers is not homogeneous. We focus on aliased registers: when an assignment to one register name can affect the value of another, such register names alias [19]. For example, Figure 1 shows the set of general purpose registers used in the $\mathrm{x} 86$ architecture. The $\mathrm{x} 86$ architecture has four general purpose 16-bit registers that can also be used as eight 8-bit registers. Each 8-bit register is called a low address or a high address. The initial bits of a 16-bit register must be aligned with the initial bits of a low-address 8-bit register. The x86 architecture allows the combination of two 8-bit registers into one 16 bit register. Another example of aliased registers is the combination of two aligned single precision floating-point registers to form one double-precision register. Examples of architectures with such aliased registers include early versions of HP PA-RISC, the Sun SPARC, and the ARM processors. For a different kind of architecture, Scholz and Eckstein [18] describe experiments with the Carmel 20xxDSP Core, which has six 40 bit accumulators that can also be used as six 32-bit registers or as twelve 16-bit aligned registers.

Architectures that allow unaligned pairing exist but are rare. Some models even allow registers wrapping around, that is, the last and the first registers in the bank combine to form one double register. An example of this type of architecture is the ARM VFP coprocessor. 
Aliased Register Allocation. We will refer to register allocation for hardware with aliased registers as aliased register allocation.

Several research groups have proposed solutions to the aliased register allocation problem. The existing approaches to aliased register allocation are based on heuristics for graph coloring $[2,3,13,15,16,19]$, on integer linear programming [12], or on partitioned Boolean quadratic optimization $[11,18]$. Integer linear programming and partitioned Boolean quadratic optimization are flexible enough to describe many architecture irregularities but lead to compile times that are worst-case exponential in the size of the input program.

Our Results. We prove that the core aliased register allocation problem with alignment restrictions is NP-complete for straight-line programs where each variable has at most one definition point. A straight-line program is a sequence of instructions without jumps. Our proof consists of three steps, from 3-SAT via a flow problem and then a coloring problem to our register allocation problem. Intuitively, the first step transforms a Boolean formula into a graph, the second step transforms the graph into an interval graph, and the third step transforms the interval graph into a program. Our coloring problem without alignment restrictions is equivalent to the shipbuilding problem; Stockmeyer proved that the shipbuilding problem is NP-complete [10, Application 9.1, p.204], although until now, to the best of our knowledge, no proof is publicly available. While we can reduce the aligned coloring problem to the unaligned coloring problem (and thereby give a proof of Stockmeyer's theorem), we have been unsuccessful in doing a reduction in the opposite direction. The aligned case is more restricted than the unaligned case; yet our result shows that the complexity of aliased register allocation in the aligned case is NP-complete.

Our result and Stockmeyer's result may be surprising because straight-line programs where each variable has at most one definition point are extremely simple. For a homogeneous bank of registers, the core register allocation problem for straight-line programs can be solved in linear time. Our results show that register aliasing is sufficient to bump the complexity to NP-complete.

Related Work. At least two other important register allocation problems are NP-complete for straight-line programs: register allocation with precolored registers [1]; and the placement of load and store instructions to transfer values to and from memory [8]. Our proof was inspired in part by a paper of Biró, Hujter, and Tuza [1] who showed how to relate a coloring problem to a flow problem. They used a flow algorithm to solve the precoloring extension problem for interval graphs. Our proof was also inspired by a paper by Even, Itai and Shamir [7] who proved NP-completeness for the multicommodity flow problem.

Rest of the Paper. In Section 2 we define our register allocation problem and in Section 3 we define a coloring problem and reduce it to the register allocation problem. In Section 4 we introduce the notion of colored flow for simple graphs, and in Section 5 we reduce the flow problem to the coloring problem. In Section 6 we show how to reduce 3-SAT to the flow problem. In Section 7 we prove our main result, and in Section 8 we give a proof of Stockmeyer's result. 
Our proof of Stockmeyer's result builds upon our main result; we are not aware of any other publicly available proof.

\section{Aliased register allocation for straight-line programs}

Programs. We will define a family of programs that compute with short values and long values. A short value can be represented with half as many bits as a long value. We use $v$ to range over program variables; a variable is either of type short or of type long. A variable of type short can only hold short values, and a variable of type long can only hold long values. We define a statement by this grammar:

$$
\begin{aligned}
& \text { (Statement) } S::=\text { short } v=(\text { definition of } v) \\
& \text { l long } v=\text { (definition of } v \text { ) } \\
& =v \quad \text { (use of } v \text { ) }
\end{aligned}
$$

A statement either defines a variable or uses a variable. We define a straight-line program to be a sequence of statements with the property that each variable is defined only once and used at least once, and every use of a variable comes after its definition.

In program $S_{1} ; \ldots ; S_{q}$, a variable $v$ is live at statement $S_{j}$, if $v$ is defined at $S_{i}, i \leq j$ and $v$ is used at $S_{k}, j<k$ [20]. Let $i$ be the index of the statement that defines $v$, and let $k$ be the index of the last statement that uses $v$. The live range of $v$ is the half open interval $[i, k[$, which includes $i$ and excludes $k$.

If $v_{1}, v_{2}$ are variables and their live ranges have a nonempty intersection, then we say that $v_{1}, v_{2}$ interfere [4].

Aliased Register Allocation. Suppose we have a machine with $2 K$ registers that each can hold a short value. The registers are called $r_{0}, \ldots, r_{2 K-1}$; we call them short registers. Suppose further that any two registers $r_{2 i}, r_{2 i+1}$, where $i \in 0 . . K-1$, can be used to hold a long value. Notice the restriction that two registers can hold a long value only if the indices are consecutive and the first index is even; we call this restriction the alignment restriction. The alignment restriction models, for example, the rule for how a programmer can use the 8bit registers on the $\mathrm{x} 86$. For example, $r_{4}, r_{5}$ can hold a long value, while $r_{7}, r_{8}$ cannot. We say that the two numbers $2 i, 2 i+1$ are aligned, and that the two registers $r_{2 i}, r_{2 i+1}$ are aligned. We use the notation that for a natural number $i, \overline{2 i}=2 i+1$ and $\overline{2 i+1}=2 i$.

We will study the problem of mapping program variables to machine registers:

CORE ALIASED REGISTER ALLOCATION WITH ALIGNMENT RESTRICTIONS (CARAAR):

Instance: a straight line program with $s$ short variables and $l$ long variables, and a number $2 K$ of available short registers $r_{0}, \ldots, r_{2 K-1}$.

Question: can each short variable be mapped to one of the short registers and can each long variable be mapped to a pair $r_{2 i}, r_{2 i+1}, i \in$ $0 . . K-1$, of short registers, such that variables with interfering live ranges are assigned registers that are all different? 


\section{Interval graphs and aligned 1-2-coloring}

Interval Graphs. We recall the definitions of an intersection graph and an interval graph [10, p.9].

Let $\mathcal{S}$ be a finite family of nonempty sets. The intersection graph of $\mathcal{S}$ is obtained by representing each set in $\mathcal{S}$ by a vertex and connecting two vertices by an edge if and only if their corresponding sets intersect. An interval graph is an intersection graph of a family of subintervals of an interval of the real numbers. We will examine weighted interval graphs for which each vertex is assigned a weight of either one or two.

For one of our programs $P$, the interference graph is the intersection graph of the family of live ranges of $P$. For each short variable, we assign the corresponding live range the weight one, and for each long variable, we assign the corresponding live range the weight two.

Aligned 1-2-Coloring. We will study a variant of graph coloring which we call aligned 1-2-coloring. We will use natural numbers as colors; for example, if we have $2 K$ colors, then we will use $0,1, \ldots, 2 K-1$ as the colors. We define a 1-2-coloring to be a mapping from vertices to colors which (1) assigns one color to each vertex of weight one and (2) assigns two consecutive colors $i, i+1$ to each vertex of weight two, such that adjacent vertices have colors that are all different. A 1-2-coloring is also known as an interval coloring [10, p.203]; we prefer to use the term 1-2-coloring to avoid confusion with the notion of interval graphs. We define an aligned 1-2-coloring to be a 1-2-coloring that assigns two aligned colors to each vertex of weight two.

Aligned 1-2-COlORING OF WEIGHTED Interval GRAPhs (A12WIG):

Instance: a weighed interval graph $G$ and a number $2 K$.

Question: does $G$ have an aligned 1-2-coloring with $2 K$ colors?

From aligned 1-2-coloring to aliased register allocation. We now present a reduction of aligned 1-2-coloring of weighted interval graphs to aliased register allocation with alignment restrictions. The key step is to show that any weighted interval graph is the interference graph of one of our straight-line programs. We first define a convenient representation of interval graphs. We say that an interval graph is program like if the intervals have startpoints and endpoints that are all different, and the startpoints and endpoints of the intervals form the set $1 . .2 q$, where $q$ is the number of intervals.

Chen [5, Lemma 3] and Saha et al. [17, p.2488] have shown how to convert an interval graph $G$ with $q$ intervals to an isomorphic program-like interval graph $\operatorname{plig}(G)$ in $O(q \log q)$ time.

From a program-like weighted interval graph $G$ with $q$ intervals, we construct a program $\operatorname{prog}(G)=S_{1} ; \ldots ; S_{2 q}$ as follows. Define

$$
\forall i \in 1 . .2 q: S_{i}= \begin{cases}\operatorname{short} v_{I}= & \text { if the interval } I \text { of weight one begins at } i \\ \text { long } v_{I}= & \text { if the interval } I \text { of weight two begins at } i \\ =v_{I} & \text { if the interval } I \text { ends at } i\end{cases}
$$


We can represent $\operatorname{prog}(G)$ as an array of instructions and then the construction of $\operatorname{prog}(G)$ takes $O(q)$ time.

Following Cormen et al. [6], we use the notation $L_{1} \leq{ }_{P} L_{2}$ to denote that $L_{1}$ is polynomial-time reducible to $L_{2}$.

Lemma 1. A12WIG $\leq_{P}$ CARAAR.

Proof. From an instance $(G, 2 K)$ of A12WIG we can construct the instance (prog $(\operatorname{plig}(G)), 2 K)$ of CARAAR. If $G$ has $q$ intervals, then we can construct $\operatorname{prog}(\operatorname{plig}(G))$ in $O(q \log q)$ time. Notice that $\operatorname{plig}(G)$ is the interference graph of $\operatorname{prog}(\operatorname{plig}(G))$ because for every interval $I$ in $\operatorname{plig}(G)$, the live range of $v_{I}$ in $\operatorname{prog}(\operatorname{plig}(G))$ is $I$. We have that $G$ has an aligned 1-2-coloring with $2 K$ colors if and only if $\operatorname{plig}(G)$ has an aligned 1-2-coloring with $2 K$ colors if and only if the interference graph of $\operatorname{prog}(\operatorname{plig}(G))$ has an aligned 1-2-coloring with $2 K$ colors if and only if the answer to CARAAR question for $(\operatorname{prog}(\operatorname{plig}(G)), 2 K)$ is true.

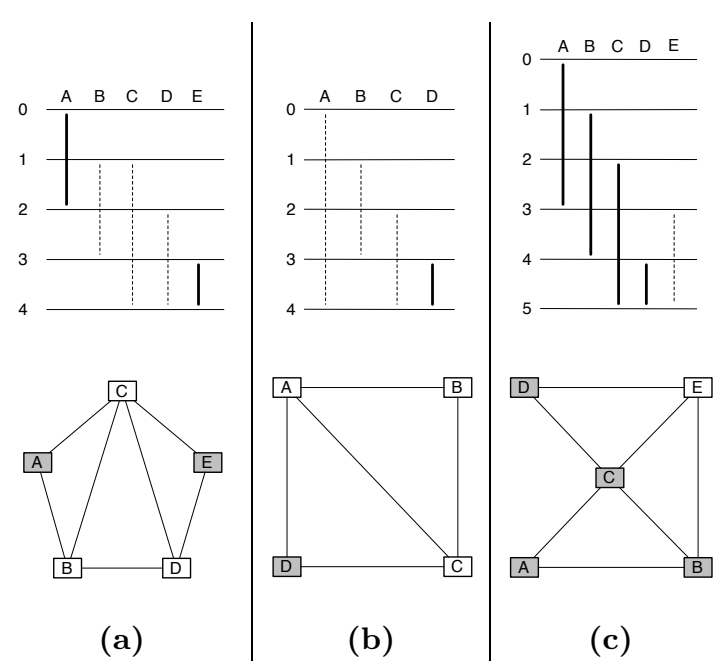

Fig. 2. Weighted interval graphs.

Example. Let us explain why aligned 1-2-coloring is a nontrivial problem. Figure 2 shows three weighted interval graphs; each graph is displayed both as a collection of intervals and in a conventional way. In the upper part of Figure 2, we use fat lines to denote intervals of weight two and we use dashed lines to denote intervals of weight one. In the lower part of Figure 2, we use shaded boxes to denote vertices of weight two, and we use white boxes to denote vertices of weight one.

A standard interval graph has the property that the size of the largest clique is equal to the minimal number of colors [10, p.17]. Aligned coloring of a weighted interval graph does not necessarily have that property. For example, 
Figure 2(a) shows a graph for which the minimal 1-2-coloring uses four colors: $A=\{0,1\}, B=2, C=3, D=0, E=\{1,2\}$, while the minimal aligned 1-2coloring uses five colors: $A=\{0,1\}, B=2, C=3, D=4, E=\{0,1\}$. Notice that the largest clique is of size 3 ; even if we treat vertices of weight two as counting as two nodes, the largest clique is of size 4.

A standard interval graph has the property that we can optimally color the graph by applying greedy coloring to any perfect elimination ordering of the vertices. (In a perfect elimination ordering, the neighbors of a node $v$ that come before $v$ in the ordering form a clique [10, p.82].) A weighted interval graph does not necessarily have that property. For example, Figure 2(b) shows a graph for which we have the perfect elimination ordering $\langle A, B, C, D\rangle$ that leads greedy coloring to produce an aligned 1-2-coloring with five colors: $A=0, B=1, C=$ $2, D=\{4,5\}$. If we drop the alignment restriction, greedy coloring again produces a 1-2-coloring with five colors: $A=0, B=1, C=2, D=\{3,4\}$. However, in both the aligned and unaligned cases, there exists an optimal assignment using just four colors: $A=0, B=2, C=1, D=\{2,3\}$.

We might try an algorithm that first applies greedy coloring to the intervals of weight one and then proceeds to color the intervals of weight two. That does not necessarily lead to an optimal 1-2-coloring. For example, Figure 2(b) shows a graph for which we have already studied the perfect elimination ordering $\langle A, B, C, D\rangle$ in which all the intervals of weight one come before the intervals of weight two. So, we will get the same suboptimal colorings as above.

Alternatively, we might try to first apply greedy coloring to the intervals of weight two, and then proceed to color the intervals of weight one. That method is not optimal either. For example, Figure 2(c) shows a graph for which the "weight-two-first" method produces the 1-2-coloring $A=\{0,1\}, B=\{2,3\}, C=$ $\{4,5\}, D=\{0,1\}, E=6$. Notice that the 1-2-coloring is also an aligned 1-2coloring. However, in both the aligned and unaligned cases, an optimal assignment uses just six colors: $A=\{0,1\}, B=\{2,3\}, C=\{4,5\}, D=\{2,3\}, E=0$.

None of the simple methods work because the aligned and unaligned 1-2coloring problems are NP-complete.

\section{Simple Graphs, Straight Cuts, and Colored Flows}

Following Cormen et al. [6], we define a network ( $V, E$, Source, Sink, $c)$ to be a directed graph with vertex set $V$, edge set $E$, distinguished vertices Source and Sink, and a capacity function $c: E \rightarrow N a t$ where $N a t$ denotes the nonnegative natural numbers, such that every vertex is on some path from Source to Sink. A cut $(S, T)$ of a network $(V, E$, Source, Sink, $c)$ is a partition of $V$ into $S$ and $T=V \backslash S$ such that Source $\in S$ and $\operatorname{Sink} \in T$. The capacity of a cut $(S, T)$, written $c(S, T)$, is given by the formula:

$$
c(S, T)=\Sigma_{(u, v) \in E, u \in S, v \in T} c(u, v)
$$

which says that the capacity of the cut is the sum of the capacities of the edges that cross the cut from $S$ to $T$. 


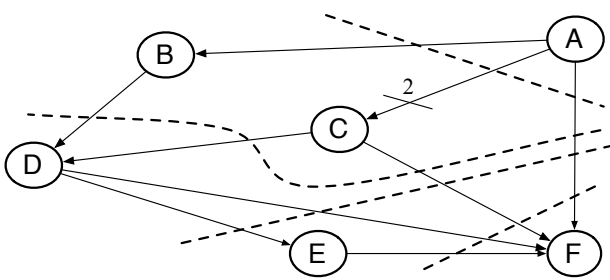

(a)

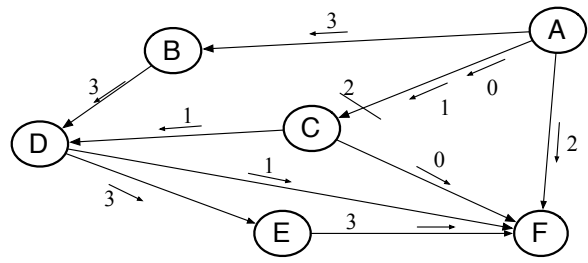

(b)

Fig. 3. (a) Simple graph; (b) colored flow.

For an acyclic network ( $V, E$, Source, Sink, c), a straight cut $(S, T)$ is a cut for which $S=\left\{v_{1}, v_{2}, \ldots, v_{i}\right\}, T=\left\{v_{i+1}, v_{i+2}, \ldots v_{n}\right\}$, and $v_{1}, \ldots, v_{n}$ is a topological ordering of $V$. A simple graph is an acyclic network for which all straight cuts have the same capacity.

Figure 3 (a) shows a simple graph. Each dashed line marks a straight cut. Each unlabeled edge has unit capacity while each remaining edge is marked with a small bar and its capacity.

Lemma 2. Let $G=(V, E$, Source, Sink, c) be an acyclic network. All straight cuts of $G$ have the same capacity if and only if $\forall v \in V \backslash\{$ Source, Sink $\}$ : $\sum_{(u, v) \in E} c(u, v)=\sum_{(v, w) \in E} c(v, w)$.

Proof. $\Rightarrow$ ) Suppose all straight cuts of $G$ have the same capacity. Let $v_{1}, \ldots, v_{n}$ be a topological ordering of $V$. Notice that $v_{1}=$ Source and $v_{n}=$ Sink. Let $i \in 2 . . n-1$. We need to show $\sum_{\left(u, v_{i}\right) \in E} c\left(u, v_{i}\right)=\sum_{\left(v_{i}, w\right) \in E} c\left(v_{i}, w\right)$. Define

$$
\begin{array}{ll}
S_{1}=\left\{v_{1}, \ldots, v_{i-1}\right\} & T_{1}=\left\{v_{i}, \ldots, v_{n}\right\} \\
S_{2}=\left\{v_{1}, \ldots, v_{i}\right\} & T_{2}=\left\{v_{i+1}, \ldots, v_{n}\right\} .
\end{array}
$$

The edges that cross the cut from $S_{1}$ to $T_{1}$ but not from $S_{2}$ to $T_{2}$ are the set of edges of the form $\left(u, v_{i}\right)$. The edges that cross the cut from $S_{2}$ to $T_{2}$ but not from $S_{1}$ to $T_{1}$ are the set of edges of the form $\left(v_{i}, w\right)$. We have that the two straight cuts $\left(S_{1}, T_{1}\right)$ and $\left(S_{2}, T_{2}\right)$ have the same capacity so $\sum_{\left(u, v_{i}\right) \in E} c\left(u, v_{i}\right)=$ $\sum_{\left(v_{i}, w\right) \in E} c\left(v_{i}, w\right)$, as desired.

$\Leftarrow)$ Suppose $\forall v \in V \backslash\{$ Source, Sink $\}: \sum_{(u, v) \in E} c(u, v)=\sum_{(v, w) \in E} c(v, w)$. We will show that for every straight cut $(S, T)$, we have $c(S, T)=c(\{$ Source $\}, V \backslash$ $\{$ Source $\})$. Let $(S, T)$ be a straight cut and let $v_{1}, \ldots, v_{n}$ be a topological ordering of $V$ such that $S=\left\{v_{1}, v_{2}, \ldots, v_{i^{\prime}}\right\}$ and $T=\left\{v_{i^{\prime}+1}, v_{i^{\prime}+2}, \ldots v_{n}\right\}$. For every $i \in 1 . . n-1$, define $S_{i}=\left\{v_{1}, v_{2}, \ldots, v_{i}\right\}, T_{i}=\left\{v_{i+1}, v_{i+2}, \ldots v_{n}\right\}$. Notice that, for every $i \in 1 . . n-1,\left(S_{i}, T_{i}\right)$ is a straight cut. Notice also that $(S, T)=\left(S_{i^{\prime}}, T_{i^{\prime}}\right)$.

Let us show by induction that for every $i \in 1 . . n-1, c\left(S_{i}, T_{i}\right)=c\left(S_{1}, T_{1}\right)$. In the case of $i=1$, the property is immediate. In the induction step, we assume $c\left(S_{i}, T_{i}\right)=c\left(S_{1}, T_{1}\right)$, and by reasoning similar to what we used on 
two straight cuts in the other half of the proof of the lemma, we have that $c\left(S_{i}, T_{i}\right)=c\left(S_{i+1}, T_{i+1}\right)$. From transitivity, we conclude that $c\left(S_{i+1}, T_{i+1}\right)=$ $c\left(S_{i}, T_{i}\right)=c\left(S_{1}, T_{1}\right)$.

Finally we have $\left(S_{1}, T_{1}\right)=(\{$ Source $\}, V \backslash\{$ Source $\})$ so every straight cut has a capacity equal to the capacity of ( $\{$ Source $\}, V \backslash\{$ Source $\})$, so all straight cuts have the same capacity.

For a network $(V, E$, Source, Sink, $c)$, a flow is a function $f: E \rightarrow$ Nat, such that

$$
\begin{array}{lr}
\forall(u, v) \in E: f(u, v) \leq c(u, v) & \text { (Capacity) } \\
\forall v \in V \backslash\{\text { Source, Sink }\}: \Sigma_{(u, v) \in E} f(u, v)=\Sigma_{(v, w) \in E} f(v, w) & \text { (Conservation) }
\end{array}
$$

For a simple graph, the capacity function is a flow. To see that, notice that the capacity function trivially satisfies the Capacity constraint and that Lemma 2 guarantees that the capacity function satisfies the Conservation constraint.

We say that an element of $0 . . K-1$ is a color. For a function $h: E \rightarrow 2^{0 . . K-1}$, the set of colors used to color edges across a cut $(S, T)$, written $h(S, T)$, is given by the formula:

$$
h(S, T)=\bigcup_{(u, v) \in E, u \in S, v \in T} h(u, v) .
$$

We define a colored flow for a simple graph $(V, E$, Source, Sink, c) with every straight cut of capacity $K$ as a function $h: E \rightarrow 2^{0 . . K-1}$ such that for every straight cut $(S, T)$, we have $h(S, T)=0 . . K-1$. Thus, for any straight cut, every color is used exactly once in the coloring of the edges that cross the cut. Every colored flow $h$ satisfies that for all $e \in E,|h(e)|=c(e)$. To see that, notice that every edge $e$ crosses some straight cut $(S, T)$ and that $|h(S, T)|=c(S, T)$. Figure 3 (b) shows an example of a colored flow.

Lemma 3. Let $G=(V, E$, Source, Sink, c) be a simple graph with every straight cut of capacity $K$. A function $h: E \rightarrow 2^{0 . . K-1}$ is a colored flow if and only if $\forall v \in V \backslash\{$ Source, Sink $\}: \cup_{(u, v) \in E} h(u, v)=\cup_{(v, w) \in E} h(v, w)$, and there exists a straight cut $(S, T)$ such that $h(S, T)=0 . . K-1$.

Proof. $\Rightarrow$ ) Suppose $h$ is a colored flow. First, let us show that $\forall v \in V \backslash\{$ Source, Sink $\}$ : $\cup_{(u, v) \in E} h(u, v)=\cup_{(v, w) \in E} h(v, w)$. Let $v_{1}, \ldots, v_{n}$ be a topological ordering of $V$. Notice that $v_{1}=$ Source and $v_{n}=$ Sink. Let $i \in 2 . . n-1$. We need to show $\cup_{\left(u, v_{i}\right) \in E} h\left(u, v_{i}\right)=\cup_{\left(v_{i}, w\right) \in E} h\left(v_{i}, w\right)$. Define

$$
\begin{array}{ll}
S_{1}=\left\{v_{1}, \ldots, v_{i-1}\right\} & T_{1}=\left\{v_{i}, \ldots, v_{n}\right\} \\
S_{2}=\left\{v_{1}, \ldots, v_{i}\right\} & T_{2}=\left\{v_{i+1}, \ldots, v_{n}\right\} .
\end{array}
$$

The edges that cross the cut from $S_{1}$ to $T_{1}$ but not from $S_{2}$ to $T_{2}$ are the set of edges of the form $\left(u, v_{i}\right)$. The edges that cross the cut from $S_{2}$ to $T_{2}$ but not from $S_{1}$ to $T_{1}$ are the set of edges of the form $\left(v_{i}, w\right)$. From $h$ being a colored flow we have that the same set of colors is used to color both the edges that cross 
$\left(S_{1}, T_{1}\right)$ and the edge that cross $\left(S_{2}, T_{2}\right)$. So, the set of colors used for edges of the form $\left(u, v_{i}\right)$ must be the same set of colors used for edges of the form $\left(v_{i}, w\right)$.

Second, for the straight cut (\{Source $\}, V \backslash\{$ Source $\}$ ) we have from $h$ being a colored flow that every color is used exactly once in the coloring of edges that cross the cut.

$\Leftarrow)$ Suppose $h$ is a function such that $\forall v \in V \backslash\{$ Source, $\operatorname{Sink}\}: \cup_{(u, v) \in E} h(u, v)=$ $\cup_{(v, w) \in E} h(v, w)$, and there exists a straight cut $(S, T)$ such that $h(S, T)=0 . . K-$ 1. We must show that for any straight cut $(S, T)$, we have $h(S, T)=0 . . K-1$. It is sufficient to show that for every straight cut $(S, T)$, we have $h(S, T)=$ $h(\{$ Source $\}, V \backslash\{$ Source $\})$. Let $(S, T)$ be a straight cut and let $v_{1}, \ldots, v_{n}$ be a topological ordering of $V$ such that $S=\left\{v_{1}, v_{2}, \ldots, v_{i^{\prime}}\right\}$ and $T=\left\{v_{i^{\prime}+1}, v_{i^{\prime}+2}, \ldots v_{n}\right\}$. For every $i \in 1 . . n-1$, define $S_{i}=\left\{v_{1}, v_{2}, \ldots, v_{i}\right\}, T_{i}=\left\{v_{i+1}, v_{i+2}, \ldots v_{n}\right\}$. Notice that, for every $i \in 1 . . n-1,\left(S_{i}, T_{i}\right)$ is a straight cut. Notice also that $(S, T)=\left(S_{i^{\prime}}, T_{i^{\prime}}\right)$.

Let us show by induction that for every $i \in 1 . . n-1, h\left(S_{i}, T_{i}\right)=h\left(S_{1}, T_{1}\right)$. In the case of $i=1$, the property is immediate. In the induction step, we assume $h\left(S_{i}, T_{i}\right)=h\left(S_{1}, T_{1}\right)$, and by reasoning similar to what we used on two straight cuts in the other half of the proof of the lemma, we have that $h\left(S_{i}, T_{i}\right)=h\left(S_{i+1}, T_{i+1}\right)$. From transitivity, we conclude that $h\left(S_{i+1}, T_{i+1}\right)=$ $h\left(S_{i}, T_{i}\right)=h\left(S_{1}, T_{1}\right)$.

Finally we have $\left(S_{1}, T_{1}\right)=(\{$ Source $\}, V \backslash\{$ Source $\})$ so the set of colors used for the edges across any given straight cut is the set same used for the edges across $(\{$ Source $\}, V \backslash\{$ Source $\})$.

Aligned colored flow. Suppose we have a simple graph (V,E, Source, Sink, c) with all straight cuts of capacity $2 K$. We define an aligned colored flow to be a colored flow $h$ such that for every $e \in E$, if $2 \leq c(e)$, then $\exists i: 0 \leq i \leq$ $K-1 \wedge\{2 i, 2 i+1\} \subseteq h(e)$. Intuitively, an edge $e$ with a capacity of at least two requires $h$ to assign $e$ aligned colors $2 i$ and $2 i+1$, among others.

For a natural number $B$, a network $(V, E$, Source, Sink, $c)$ is $B$-bounded if for all $e \in E: c(e) \leq B$.

Aligned COLORED Flow (ACFlow):

Instance: a 6-bounded simple graph $G$.

Question: does $G$ have an aligned colored flow?

It is straightforward to see that ACFLOw is in NP. Our reduction from 3-SAT to ACFLOW (Section 6) produces a 6-bounded simple graph.

\section{From aligned colored flow to aligned 1-2-coloring}

In this section we present a reduction of the aligned colored flow problem to aligned 1-2-coloring of weighted interval graphs.

Lemma 4. ACFLOW $\leq_{P}$ A12wiG. 
Proof. Let $G=(V, E$, Source, Sink, $c)$ be an instance of ACFLow, and let $2 K$ be the capacity of all straight cuts of $G$. From $G$ we construct a weighted interval graph $\operatorname{wig}(G)$ in the following way. Let $v_{1}, \ldots, v_{n}$ be a topological ordering of $V$. The intervals of wig $(G)$ are defined as follows. For each $\left(v_{i}, v_{j}\right) \in E$ such that $2 \leq c\left(v_{i}, v_{j}\right)$, we create one interval $\left[i, j\left[\right.\right.$ of weight two, and we create $c\left(v_{i}, v_{j}\right)-2$ intervals $\left[i, j\left[\right.\right.$ of weight one. For each $\left(v_{i}, v_{j}\right) \in E$ such that $c\left(v_{i}, v_{j}\right)=1$, we create one interval $[i, j[$ of weight one.

From $G$ we can construct the instance (wig $(G), 2 K$ ) of A12wig. We can construct the topological ordering of $V$ in $O(|V|+|E|)$ time and we can construct the intervals of $\operatorname{wig}(G)$ in $O(|E|)$ time, so we can construct (wig $(G), 2 K$ ) in $O(|V|+|E|)$ time.

We will show that $G$ has an aligned colored flow if and only if wig $(G)$ has an aligned 1-2-coloring with $2 K$ colors.

$\Rightarrow)$ Suppose $G$ has an aligned colored flow $h$. We can then define a mapping $\kappa$ that assigns colors to each vertex of $\operatorname{wig}(G)$ as follows. For each $\left(v_{i}, v_{j}\right) \in E$ such that $2 \leq c\left(v_{i}, v_{j}\right)$, we have that $h\left(v_{i}, v_{j}\right)$ contains two aligned colors; assign those two colors to the interval of weight two created from $\left(v_{i}, v_{j}\right)$, and assign each of the rest of the colors in $h\left(v_{i}, v_{j}\right)$ to each of the intervals of weight one created from $\left(v_{i}, v_{j}\right)$. For each $\left(v_{i}, v_{j}\right) \in E$ such that $c\left(v_{i}, v_{j}\right)=1$, assign the one color in $h\left(v_{i}, v_{j}\right)$ to the interval of weight one created from $\left(v_{i}, v_{j}\right)$. We need to show that adjacent vertices in wig $(G)$ have colors that are all different. Suppose we have two adjacent intervals $I_{1}, I_{2}$ in wig $(G)$, that is, they have a nonempty intersection. Since all the intervals are half-open, the intersection consists of more than one point. Choose a point $p$ in the intersection which is not the start or end point of any interval in $\operatorname{wig}(G)$. Define $S=\left\{v_{i} \in V \mid i<p\right\}$ and define $T=\left\{v_{i} \in V \mid i>p\right\}$. We have that $(S, T)$ is a straight cut. We also have that $I_{1}, I_{2}$ both cross the cut. For every straight cut, every color is used exactly once, so $I_{1}, I_{2}$ have colors that are all different.

$\Leftrightarrow)$ Suppose wig $(G)$ has an aligned 1-2-coloring $\kappa$. We can then define a mapping $h: E \rightarrow 2^{0.2 K-1}$ as follows. For each $\left(v_{i}, v_{j}\right) \in E$, let $h\left(v_{i}, v_{j}\right)$ be the union of the colors assigned by $\kappa$ to the intervals in wig $(G)$ created from $\left(v_{i}, v_{j}\right)$. We need to show that $h$ is an aligned colored flow.

Let us first show that $h$ is a colored flow. From Lemma 3 we have that we must show that (1) $\forall v \in V \backslash\{$ Source, Sink $\}: \cup_{(u, v) \in E} h(u, v)=\cup_{(v, w) \in E} h(v, w)$, and (2) there exists a straight cut $(S, T)$ such that $\cup_{(u, v) \in E, u \in S, v \in T} h(u, v)=$ $0 . .2 K-1$.

To prove (1), let $v_{j}$ be a vertex in $V \backslash\{$ Source, Sink $\}$. We have that

$$
\begin{aligned}
& C_{1}=\left(\left\{v_{i} \mid i<j\right\},\left\{v_{k} \mid j \leq k\right\}\right) \\
& C_{2}=\left(\left\{v_{i} \mid i \leq j\right\},\left\{v_{k} \mid j<k\right\}\right)
\end{aligned}
$$

are straight cuts of $G$ and hence both of capacity $2 K$. Next define $E_{1}$ to be the set of edges in $E$ of the form $\left(v_{i}, v_{j}\right)$, and define $E_{2}$ to be the set of edges in $E$ of the form $\left(v_{j}, v_{k}\right)$. From Lemma 2 we have that $\Sigma_{e \in E_{1}} c(e)=\Sigma_{e \in E_{2}} c(e)$. We can find a subset $E^{\prime} \subseteq E$ such that $E^{\prime} \cap E_{1}=\emptyset, E^{\prime} \cap E_{2}=\emptyset$, the edges that cross $C_{1}$ can be written $E_{1} \cup E^{\prime}$, and the edges that cross $C_{2}$ can be written 
$E_{2} \cup E^{\prime}$. We conclude that $\cup_{e \in E_{1}} h(e)=\cup_{e \in E_{2}} h(e)$, as required. To prove (2), let us examine the edges that traverse the straight cut ( $\{$ Source $\}, V \backslash\{$ Source $\}$ ), which by assumption has capacity $2 K$. From those edges, we create intervals in wig $(G)$ that all overlap and hence get all different colors by $\kappa$.

Finally, we have that $h$ is aligned because the construction of $\operatorname{wig}(G)$ ensures that for any $e$ for which we have $2 \leq c(e)$, one vertex of weight two is created; the construction of $h$ then ensures that the aligned colors assigned by $\kappa$ to that interval will be two of the colors assigned by $h$ to $e$.

\section{From 3-SAT to aligned colored flow}

The 3-SAT problem is to decide whether a given Boolean formula in conjunctive normal form $(\mathrm{CNF})$ is satisfiable. In this section we present a reduction of 3-SAT to the aligned colored flow problem. Let

$$
\begin{aligned}
F & =\wedge_{j=1}^{m} c_{j} \\
c_{j} & =l_{j 1} \vee l_{j 2} \vee l_{j 3}
\end{aligned}
$$

be a formula with $n$ Boolean variables $x_{1}, \ldots, x_{n}$ and $m$ clauses $c_{1}, \ldots, c_{m}$; each literal, $l_{j 1}$ or $l_{j 2}$ or $l_{j 3}$, is either a variable or the negation of a variable, and in each clause the three literals are distinct. Let $p_{i}$ be the number of occurrences of $x_{i}$, and let $q_{i}$ be the number of occurrences of $\bar{x}_{i}$. For convenience, we define $p_{0}=0$ and $q_{0}=0$.

From a Boolean formula $F$ in CNF we construct a simple graph $\operatorname{sg}(F)=$ $(V, E$, Source, Sink, $c)$. The graph is akin to the graph used by Even, Itai and Shamir [7, Section 4] in their proof of NP-completeness for the multicommodity flow problem. The vertices of $\operatorname{sg}(F)$ are:

$$
\begin{aligned}
V= & \{\text { Source, Sink, } Q, R\} \cup \\
& \left\{c_{j} \mid 1 \leq j \leq m\right\} \cup \\
& \left\{a_{i}, b_{i} \mid 1 \leq i \leq n\right\} \cup \\
& \left\{s_{i 0}, s_{i y}, s_{i\left(p_{i}+1\right)}, x_{i y}, x_{i\left(p_{i}+1\right)} \mid 1 \leq i \leq n \wedge 1 \leq y \leq p_{i}\right\} \cup \\
& \left\{\quad \bar{s}_{i z}, \bar{s}_{i\left(q_{i}+1\right)}, \bar{x}_{i z}, \bar{x}_{i\left(q_{i}+1\right)} \mid 1 \leq i \leq n \wedge 1 \leq z \leq q_{i}\right\}
\end{aligned}
$$

For convenience, we will some times use the alias $a_{n+1}$ for $R$. Figure 4 shows a listing of the edges in $E$ and their capacities. Figure 4 also shows a set of colors for each edge, using the abbreviations in Figure 5(a); we will need that later.

Figure 6 illustrates the graph constructed from the formula $\left(x_{1} \vee x_{2} \vee x_{3}\right) \wedge$ $\left(\bar{x}_{1} \vee x_{2} \vee \bar{x}_{3}\right)$. We use $c_{1}$ to denote $\left(x_{1} \vee x_{2} \vee x_{3}\right)$ and we use $c_{2}$ to denote $\left(\bar{x}_{1} \vee x_{2} \vee \bar{x}_{3}\right)$. Let us now give an intuitive explanation of the construction of the graph. For each variable $x_{i}$, we construct a lobe. First we add the edges $\left(x_{i y} \stackrel{1}{\rightarrow} x_{i(y+1)}\right)$ to form an upper path and we add the edges $\left(\bar{x}_{i z} \stackrel{1}{\rightarrow} \bar{x}_{i(z+1)}\right)$ to form a lower path. Then we connect theses paths to $a_{i}$ and $b_{i}$ to form the lobe by adding the edges $\left(a_{i} \stackrel{1}{\rightarrow} x_{i 1}\right),\left(a_{i} \stackrel{1}{\rightarrow} \bar{x}_{i 1}\right),\left(x_{i\left(p_{i}+1\right)} \stackrel{1}{\rightarrow} b_{i}\right)$, and $\left(\bar{x}_{i\left(q_{i}+1\right)} \stackrel{1}{\rightarrow} b_{i}\right)$. 
Next we are going to make several edges that have alignment requirements. For each $s_{i y}$, we create an edge $\left(\right.$ Source $\left.\stackrel{2}{\rightarrow} s_{i y}\right)$ with a capacity two. Likewise for all the $s_{i 0}$ and the $\bar{s}_{i h}$ vertices. For each $s_{i\left(p_{i}+1\right)}$ and $\bar{s}_{i\left(q_{i}+1\right)}$ we add the edges $\left(\right.$ Source $\left.\stackrel{2}{\rightarrow} s_{i\left(p_{i}+1\right)}\right)$ and (Source $\stackrel{2}{\longrightarrow} \bar{s}_{i\left(q_{i}+1\right)}$ ) each with capacity two. Next we will add an edge (Source $\stackrel{2}{\rightarrow} Q$ ) also with capacity of two. In total we have made a capacity of $2(3 m+3 n+1)$ leaving the source. We want to make $\operatorname{sg}(F)$ simple, so there must be capacities of two leaving each of these vertices and eventually reaching the Sink. We will create some more aligned edges which will now connect certain vertices to Sink. For each of the $c_{j}$ vertices, we create the edges $\left(c_{j} \stackrel{6}{\rightarrow} \operatorname{Sink}\right)$ with a capacity of six and finally we add $(R \stackrel{2}{\rightarrow}$ Sink $)$ with a capacity of two. Now all that remains to make the graph simple is to connect the $Q, s_{i y}, s_{i\left(p_{i}+1\right)}, \bar{s}_{i z}$ and $\bar{s}_{i\left(q_{i}+1\right)}$ vertices to $R, c_{j}$, and Sink.

We will first add edges to send the current excess capacity at $Q$ to $R$. We will add a direct edge $(Q \stackrel{1}{\rightarrow} R)$ to get one unit of capacity to $R$. To get the other unit to $R$, we will connect the lobes serially, by adding the edges $\left(b_{i} \stackrel{1}{\rightarrow} a_{i+1}\right)$. Finally, we add $\left(Q \stackrel{1}{\rightarrow} a_{1}\right)$, resulting in a path to send the other unit of capacity to $R$ and two units of capacity reaching Sink.

The $a_{i}$ and $b_{i}$ vertices still have an imbalance of capacity and must have edges to supply capacity or drain it. To correct for these imbalances, we add the edges $\left(s_{i 0} \stackrel{1}{\rightarrow} \operatorname{Sink}\right),\left(s_{i 0} \stackrel{1}{\rightarrow} a_{i}\right)$, and $\left(b_{i} \stackrel{1}{\rightarrow} \operatorname{Sink}\right)$. This results in a current total of $2 n+2$ units of capacity reaching Sink, and that the vertices on the lobe are balanced.

We will now connect the remaining $s_{i y}, s_{i\left(p_{i}+1\right)}, \bar{s}_{i z}$ and $\bar{s}_{i\left(q_{i}+1\right)}$ vertices to the $c_{j}$ vertices and Sink. We add the edges $\left(s_{i y} \stackrel{\overrightarrow{1}}{\rightarrow} x_{i y}\right),\left(s_{i\left(p_{i}+1\right)} \stackrel{1}{\rightarrow} x_{i\left(p_{i}+1\right)}\right),\left(\bar{s}_{i z} \stackrel{1}{\rightarrow}\right.$ $\left.x_{i z}\right)$ and $\left(\bar{s}_{i\left(q_{i}+1\right)} \stackrel{1}{\rightarrow} \bar{x}_{i h}\right)$ which will send one unit of capacity from each of these vertices to the corresponding vertices on the lobe. The other units from the $s_{i y}$ and $\bar{s}_{i z}$ vertices will be sent to some $c_{j}$ vertex while those of $s_{i\left(p_{i}+1\right)}$ and $\bar{s}_{i\left(p_{i}+1\right)}$ will be sent directly to Sink. We add the edges $\left(s_{i\left(p_{i}+1\right)} \stackrel{1}{\rightarrow} \operatorname{Sink}\right)$ and $\left(\bar{s}_{i\left(q_{i}+1\right)} \stackrel{1}{\rightarrow}\right.$ Sink $)$, which now results in an additional $2 n$ units of capacity reaching Sink for a running total of $4 n+2$. For the remaining vertices, we add an edge $\left(s_{i y} \stackrel{1}{\rightarrow} c_{j}\right)$ if the $y^{t h}$ occurrence of $x_{i}$ appears in $c_{j}$. For the $\bar{s}_{i z}$ vertices, we add similar edges. From these edges we get $3 m$ units of capacity reaching Sink, because each of these edges corresponds to a clause, and each clause has exactly three literals in it. All that remains is to drain the single unit of capacity currently residing at the $x_{i y}, x_{i\left(p_{i}+1\right)}, \bar{x}_{i z}$ and $\bar{x}_{i\left(q_{i}+1\right)}$ vertices and we will have a simple graph. We add the edges $\left(x_{i(y+1)} \stackrel{1}{\rightarrow} c_{j}\right)$ if the $y^{\text {th }}$ appearance of $x_{i}$ occurs in $c_{j}$ as well as $\left(x_{i(z+1)} \stackrel{1}{\rightarrow} c_{j}\right)$ if the $z^{\text {th }}$ appearance of $\bar{x}_{i}$ occurs in $c_{j}$. This results in another $3 m$ units of capacity reaching Sink. Finally, the last $2 n$ units will be supplied by the edges $\left(x_{i 1} \stackrel{1}{\rightarrow} \operatorname{Sink}\right)$ and $\left(\bar{x}_{i 1} \stackrel{1}{\rightarrow} \operatorname{Sink}\right)$. 


\begin{tabular}{|c|c|c|c|c|c|}
\hline & Edge & Color & & Edge & Color \\
\hline 1 & $x_{i y} \stackrel{1}{\rightarrow} x_{i(y+1)}$ & $\psi\left(x_{i}\right) ? \alpha(i, y): \theta$ & 18 & $s_{i 0} \stackrel{1}{\rightarrow}$ Sink & $\overline{\gamma(i)}$ \\
\hline 2 & $\bar{x}_{i z} \stackrel{1}{\rightarrow} \bar{x}_{i(z+1)}$ & $\psi\left(x_{i}\right) ? \theta: \beta(i, z)$ & 19 & $s_{i 0} \stackrel{1}{\rightarrow} a_{i}$ & $\gamma(i)$ \\
\hline 3 & $a_{i} \stackrel{1}{\rightarrow} x_{i 1}$ & $\psi\left(x_{i}\right) ? \gamma(i): \theta$ & 20 & $b_{i} \stackrel{1}{\rightarrow} \operatorname{Sink}$ & $\psi\left(x_{i}\right) ? \delta(i): \eta(i)$ \\
\hline 4 & $a_{i} \stackrel{1}{\rightarrow} \bar{x}_{i 1}$ & $\psi\left(x_{i}\right) ? \theta: \gamma(i)$ & 21 & $s_{i y} \stackrel{1}{\rightarrow} x_{i y}$ & $\alpha(i, y)$ \\
\hline 5 & $x_{i\left(p_{i}+1\right)} \stackrel{1}{\rightarrow} b_{i}$ & $\psi\left(x_{i}\right) ? \delta(i): \theta$ & 22 & $s_{i\left(p_{i}+1\right)} \stackrel{1}{\rightarrow} x_{i\left(p_{i}+1\right)}$ & $\delta(i)$ \\
\hline 6 & $\bar{x}_{i\left(q_{i}+1\right)} \stackrel{1}{\rightarrow} b_{i}$ & $\psi\left(x_{i}\right) ? \theta: \eta(i)$ & 23 & $\bar{s}_{i z} \stackrel{1}{\rightarrow} \bar{x}_{i z}$ & $\beta(i, z)$ \\
\hline 7 & Source $\stackrel{2}{\rightarrow} s_{i y}$ & $\overline{\alpha(i, y), \overline{\alpha(i, y)}}$ & 24 & $\bar{s}_{i\left(q_{i}+1\right)} \stackrel{1}{\rightarrow} \bar{x}_{i\left(q_{i}+1\right)}$ & $\eta(i)$ \\
\hline 8 & Source $\stackrel{2}{\rightarrow} s_{i\left(p_{i}+1\right)}$ & $\overline{\delta(i), \overline{\delta(i)}}$ & 25 & $s_{i\left(p_{i}+1\right)} \stackrel{1}{\rightarrow} \operatorname{Sink}$ & $\overline{\delta(i)}$ \\
\hline 9 & Source $\stackrel{2}{\rightarrow} \bar{s}_{i z}$ & $\overline{\beta(i, z), \overline{\beta(i, z)}}$ & 26 & $\bar{s}_{i\left(q_{i}+1\right)} \stackrel{1}{\rightarrow} \operatorname{Sink}$ & $\overline{\eta(i)}$ \\
\hline 10 & Source $\stackrel{2}{\rightarrow} \bar{s}_{i\left(q_{i}+1\right)}$ & $\overline{\eta(i), \overline{\eta(i)}}$ & 27 & $\begin{array}{l}s_{i y} \stackrel{1}{\rightarrow} c_{j} \\
\text { if occ }\left(y, x_{i}, c_{j}\right)\end{array}$ & $\overline{\alpha(i, y)}$ \\
\hline 11 & Source $\stackrel{2}{\rightarrow} s_{i 0}$ & $\overline{\gamma(i), \overline{\gamma(i)}}$ & 28 & $\begin{array}{l}\bar{s}_{i z} \stackrel{1}{\rightarrow} c_{j} \\
\text { if } \operatorname{occ}\left(z, \bar{x}_{i}, c_{j}\right)\end{array}$ & $\overline{\beta(i, z)}$ \\
\hline 12 & Source $\stackrel{2}{\rightarrow} Q$ & $\theta, \bar{\theta}$ & 29 & $\begin{array}{l}x_{i(y+1)} \stackrel{1}{\rightarrow} c_{j} \\
\text { if } \operatorname{occ}\left(y, x_{i}, c_{j}\right),\left(y \neq p_{i}\right)\end{array}$ & $\psi\left(x_{i}\right) ? \alpha(i, y): \alpha(i, y+1)$ \\
\hline 13 & $c_{j} \stackrel{6}{\rightarrow}$ Sink & See Figure 5(b) & 30 & $\begin{array}{l}x_{i\left(p_{i}+1\right)} \stackrel{1}{\rightarrow} c_{j} \\
\text { if } \operatorname{occ}\left(p_{i}, x_{i}, c_{j}\right)\end{array}$ & $\psi\left(x_{i}\right) ? \alpha\left(i, p_{i}\right): \delta(i)$ \\
\hline 14 & $R \stackrel{2}{\rightarrow} \operatorname{Sink}$ & $\theta, \bar{\theta}$ & 31 & $\begin{array}{l}\bar{x}_{i(z+1)} \stackrel{1}{\rightarrow} c_{j} \\
\text { if } \operatorname{occ}\left(z, \bar{x}_{i}, c_{j}\right),\left(z \neq q_{i}\right)\end{array}$ & $\psi\left(x_{i}\right) ? \beta(i, z+1): \beta(i, z)$ \\
\hline 15 & $Q \stackrel{1}{\rightarrow} R$ & $\bar{\theta}$ & 32 & $\begin{array}{l}\bar{x}_{i\left(q_{i}+1\right)} \stackrel{1}{\rightarrow} c_{j} \\
\text { if } \operatorname{occ}\left(q_{i}, \bar{x}_{i}, c_{j}\right)\end{array}$ & $\psi\left(x_{i}\right) ? \eta(i): \beta\left(i, q_{i}\right)$ \\
\hline 16 & $b_{i} \stackrel{1}{\rightarrow} a_{i+1}$ & $\theta$ & 33 & $x_{i 1} \stackrel{1}{\rightarrow} \operatorname{Sink}$ & $\psi\left(x_{i}\right) ? \gamma(i): \alpha(i, 1)$ \\
\hline 17 & $Q \stackrel{1}{\rightarrow} a_{1}$ & $\theta$ & 34 & $\bar{x}_{i 1} \stackrel{1}{\rightarrow}$ Sink & $\psi\left(x_{i}\right) ? \beta(i, 1): \gamma(i)$ \\
\hline
\end{tabular}

Fig. 4. The edges of $\operatorname{sg}(F)$. We have $1 \leq i \leq n, 1 \leq j \leq m, 1 \leq y \leq p_{i}, 1 \leq z \leq q_{i}$. The notation $\psi\left(x_{i}\right) ? d_{T}: d_{F}$ denotes that if $\psi\left(x_{i}\right)=$ true then the assigned color is $d_{T}$, and if $\psi\left(x_{i}\right)=$ false then the assigned color is $d_{F}$. An expression $\operatorname{occ}\left(y, x_{i}, c_{j}\right)$ means that the $y^{\text {th }}$ occurrence of $x_{i}$ appears in $c_{j}$.

$$
\begin{aligned}
\alpha(i, y) & =2\left(\sum_{j=1}^{i} p_{j-1}+y-1\right) \\
\beta(i, z)= & 2\left(\Sigma_{j=1}^{n} p_{j}+\Sigma_{j=1}^{i} q_{j-1}\right. \\
& +z-1) \\
\gamma(i)= & 2(3 m+i-1) \\
\delta(i)= & 2(3 m+n+i-1) \\
\eta(i)= & 2(3 m+2 n+i-1) \\
\theta= & 2(3 m+3 n)
\end{aligned}
$$

\begin{tabular}{||l|l|c||}
\hline & Condition & Colors \\
\hline$i$ & $\operatorname{occ}\left(y, x_{i}, c_{j}\right) \wedge \psi\left(x_{i}\right)=$ true & $\alpha(i, y), \overline{\alpha(i, y)}$ \\
\hline$i i$ & $\operatorname{occ}\left(y, x_{i}, c_{j}\right) \wedge \psi\left(x_{i}\right)=$ false $\wedge y \neq p_{i}$ & $\alpha(i, y+1), \overline{\alpha(i, y)}$ \\
\hline$i i i$ & $\operatorname{occ}\left(p_{i}, x_{i}, c_{j}\right) \wedge \psi\left(x_{i}\right)=$ false & $\delta(i), \overline{\alpha\left(i, p_{i}\right)}$ \\
\hline$i v$ & $\operatorname{occ}\left(z, \bar{x}_{i}, c_{j}\right) \wedge \psi\left(x_{i}\right)=$ false & $\beta(i, z), \overline{\beta(i, z)}$ \\
\hline$v$ & $\operatorname{occ}\left(z, \bar{x}_{i}, c_{j}\right) \wedge \psi\left(x_{i}\right)=$ true $\wedge \neq q_{i}$ & $\beta(i, z+1), \overline{\beta(i, z)}$ \\
\hline$v i$ & $\operatorname{occ}\left(q_{i}, \bar{x}_{i}, c_{j}\right) \wedge \psi\left(x_{i}\right)=$ true & $\eta(i), \overline{\beta\left(i, q_{i}\right)}$ \\
\hline
\end{tabular}

Fig. 5. (a) Abbreviations. (b) For each literal in $c_{j}$, the two colors in $h\left(c_{j} \stackrel{6}{\rightarrow} \operatorname{Sink}\right)$. 


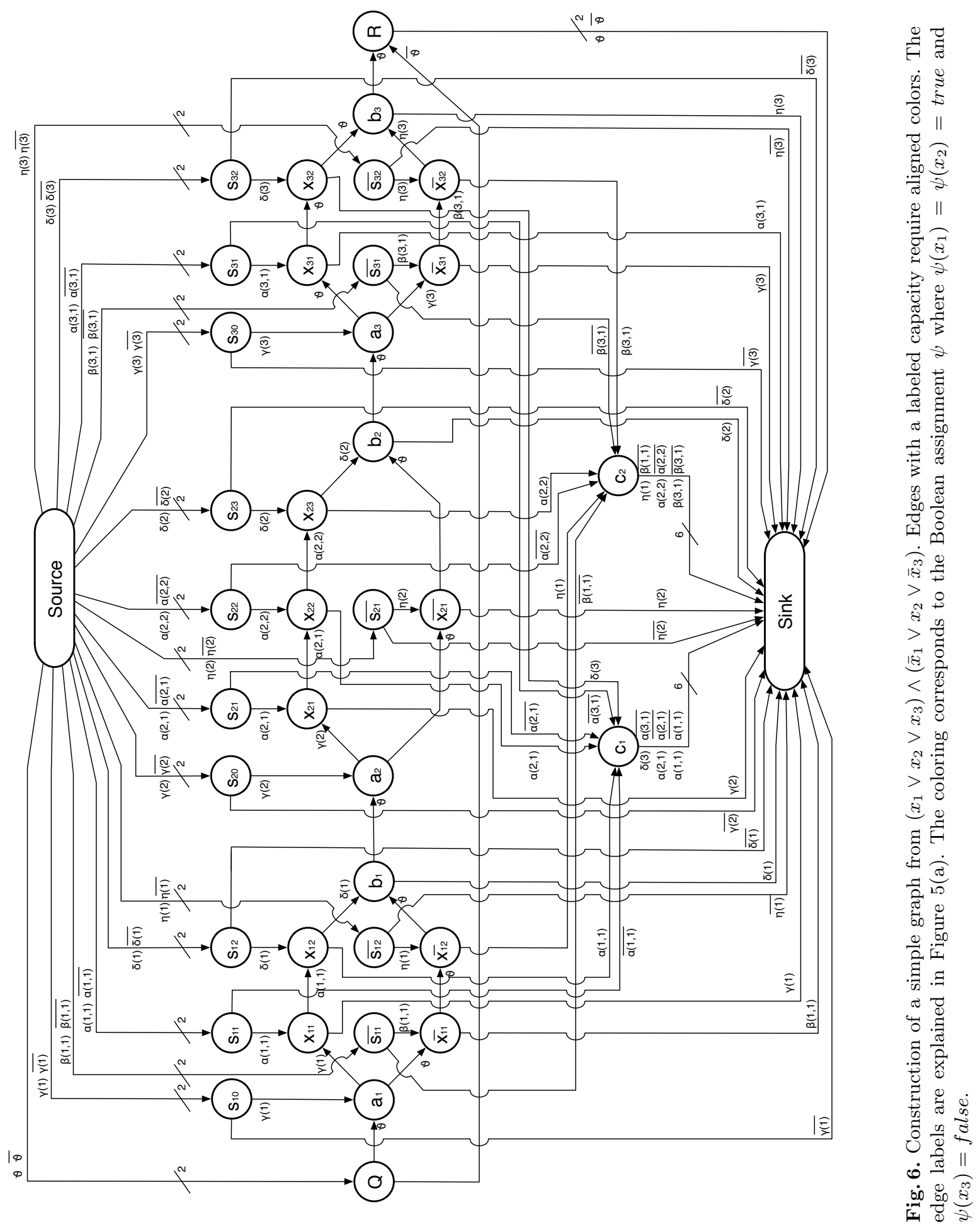


Lemma 5. 3-SAT $\leq_{P}$ ACFLOW.

Proof. From an instance $F$ of 3 -SAT with $n$ variables and $m$ clauses, we can construct the instance $\operatorname{sg}(F)$ of ACFLOw. The graph $\operatorname{sg}(F)$ has $7 m+7 n+4$ vertices and $16 m+17 n+4$ edges and we can construct $\operatorname{sg}(F)$ in polynomial time. It is straightforward to check that $\operatorname{sg}(F)$ is a 6-bounded acyclic network. We must also show that all straight cuts of $\operatorname{sg}(F)$ have the same capacity. From Lemma 2 we have that we must show $\forall v \in V \backslash\{$ Source, Sink $\}$ : $\sum_{(u, v) \in E} c(u, v)=\sum_{(v, w) \in E} c(v, w)$. We will use the terminology that for a vertex $v \in V \backslash\{$ Source, Sink $\}$, the in-capacity of $v$ is $\sum_{(u, v) \in E} c(u, v)$, and the out-capacity of $v$ is $\sum_{(v, w) \in E} c(v, w)$. We will examine each vertex in $V \backslash$ $\{$ Source, Sink\} in turn. From Figure 4 we see that, for $1 \leq i \leq n, 1 \leq j \leq m$, $1 \leq y \leq p_{i}, 1 \leq z \leq q_{i}$ :

\begin{tabular}{c|cc|cc} 
Node & in-capacity using rules & out-capacity using rules \\
\hline$Q$ & 2 & 12 & 2 & 15,17 \\
$R$ & 2 & 15,16 & 2 & 14 \\
$c_{j}$ & 6 & $27-32$ & 6 & 13 \\
$a_{i}$ & 2 & $16,17,19$ & 2 & 3,4 \\
$b_{i}$ & 2 & 5,6 & 2 & 16,20 \\
$s_{i 0}$ & 2 & 11 & 2 & 18,19 \\
$s_{i y}$ & 2 & 7 & 2 & 21,27 \\
$s_{i\left(p_{i}+1\right)}$ & 2 & 8 & 2 & 22,25 \\
$x_{i y}$ & 2 & $1,3,21$ & 2 & $1,29,33$ \\
$x_{i\left(p_{i}+1\right)}$ & 2 & 1,22 & 2 & 5,30 \\
$\bar{s}_{i z}$ & 2 & 9 & 2 & 23,28 \\
$\bar{s}_{i\left(q_{i}+1\right)}$ & 2 & 10 & 2 & 24,26 \\
$\bar{x}_{i z}$ & 2 & $1,2,23$ & 2 & $2,31,34$ \\
$\bar{x}_{i\left(q_{i}+1\right)}$ & 2 & 2,24 & 2 & 6,32 \\
\hline
\end{tabular}

So, $\operatorname{sg}(F)$ is a simple graph. flow.

We will show that $F$ is satisfiable if and only if $\operatorname{sg}(F)$ has an aligned colored

Define $K=3 m+3 n+1$.

$\Rightarrow$ ) Suppose we have a Boolean assignment $\psi$ for the variables in $F$ which satisfies $F$. Let $h: E \rightarrow 2^{0.2 K-1}$ be defined by mapping each edge to the set of colors given in Figure 4, using the abbreviations in Figure 5(a). We need to show that $h$ is an aligned colored flow.

From Lemma 3 we have that we can show that $h$ is a colored flow by showing that: (1) $\forall v \in V \backslash\{$ Source, Sink $\}: \cup_{(u, v) \in E} h(u, v)=\cup_{(v, w) \in E} h(v, w)$, and (2) there exists a straight cut $(S, T)$ such that $\cup_{(u, v) \in E, u \in S, v \in T} h(u, v)=0 . .2 K-1$.

To prove (1), we first note that it is straightforward to check that the sets of edges and colors defined in Figure 4 are the same as the sets of edges and colors shown in Figures 8, 9, and 10 in the Appendix. Then, for each vertex $v \in V \backslash\{$ Source, Sink $\}$, we examine the corresponding illustration in one of the figures Figures 8, 9, and 10, and in each case it is straightforward to check that $\cup_{(u, v) \in E} h(u, v)=\cup_{(v, w) \in E} h(v, w)$. 
To prove (2), we will focus on the straight cut (Source, $V \backslash$ Source) and show that $\cup_{(u, v) \in E, u \in \text { Source, } v \in V \backslash \text { Source }} h(u, v)=0 . .2 K-1$. From Figure 4, rules 7-12, we can see that six forms of edges cross the straight cut. Notice that $\sum_{i=1}^{n} p_{i}+$ $\sum_{i=1}^{n} q_{i}=3 \mathrm{~m}$. From that observation and the abbreviations in Figure 5(a), it is straightforward to see that the colors of those edges are:

$$
\begin{aligned}
\bigcup\left\{\alpha(i, y), \overline{\alpha(i, y)} \mid 1 \leq i \leq n \wedge 1 \leq y \leq p_{i}\right\} & =\left(0 \ldots 2\left(\sum_{i=1}^{n} p_{i}\right)-1\right) \\
\bigcup\left\{\beta(i, z), \overline{\beta(i, z)} \mid 1 \leq i \leq n \wedge 1 \leq z \leq q_{i}\right\} & =\left(2\left(\sum_{i=1}^{n} p_{i}\right) . .6 m-1\right) \\
\bigcup\{\gamma(i), \overline{\gamma(i)} \mid 1 \leq i \leq n\} & =(6 m . .6 m+2 n-1) \\
\bigcup\{\delta(i), \overline{\delta(i)} \mid 1 \leq i \leq n\} & =(6 m+2 n . .6 m+4 n-1) \\
\bigcup\{\eta(i), \overline{\eta(i)} \mid 1 \leq i \leq n\} & =(6 m+4 n . .6 m+6 n-1) \\
\{\delta, \bar{\delta}\} & =(6 m+6 n . .6 m+6 n+1)
\end{aligned}
$$

We conclude that the colors of the edges that cross the straight cut (Source, $V \backslash$ Source) form the interval $0 . .6 m+6 n+1=0 . .2 K-1$.

Finally we must show that $h$ is an aligned colored flow, that is, we must show that for every edge $e$, if $2 \leq c(e)$, then $\exists i: 0 \leq i \leq K-1 \wedge\{2 i, 2 i+1\} \subseteq h(e)$. We can easily verify that by inspection of Figure 4 .

$\Leftarrow)$ Suppose $\operatorname{sg}(F)$ has an aligned colored flow $h: E \rightarrow 2^{0.2 K-1}$. We can then define a Boolean assignment $\psi$ for the variables of $F$ :

$$
\text { for all } i \in 1 . . n: \psi\left(x_{i}\right)=\left\{\begin{array}{l}
\text { true if } h(\text { Source } \stackrel{2}{\rightarrow} Q)=h(Q \stackrel{1}{\rightarrow} R) \cup h\left(a_{i} \stackrel{1}{\rightarrow} \bar{x}_{i 1}\right) \\
\text { false otherwise }
\end{array}\right.
$$

Let $j \in 1$..m. We will show that $\psi$ satisfies $c_{j}$. Let us first prove a property of $h$ :

- Claim 1: Either there exists $i, y$ such that $h\left(\right.$ Source $\left.\stackrel{2}{\rightarrow} s_{i y}\right) \subseteq h\left(c_{j} \stackrel{6}{\rightarrow} \operatorname{Sink}\right)$, or there exists $i, z$ such that $h\left(\right.$ Source $\left.\stackrel{2}{\rightarrow} \bar{s}_{i z}\right) \subseteq h\left(c_{j} \stackrel{6}{\rightarrow}\right.$ Sink $)$.

Proof of Claim 1 . The set $h\left(c_{j} \stackrel{6}{\rightarrow} \operatorname{Sink}\right)$ contains two aligned colors $d, \bar{d}$. The edges crossing the straight cut $(\{$ Source $\}, V \backslash\{$ Source $\})$ are of the six forms: (i) Source $\stackrel{2}{\rightarrow} Q$, (ii) Source $\stackrel{2}{\rightarrow} s_{i 0}$, (iii) Source $\stackrel{2}{\rightarrow} s_{i\left(p_{i}+1\right)}$, (iv) Source $\stackrel{2}{\rightarrow} \bar{s}_{i\left(q_{i}+1\right)}$, (v) Source $\stackrel{2}{\rightarrow} s_{i y}$, (vi) Source $\stackrel{2}{\rightarrow} \bar{s}_{i z}$. Because every color is used exactly once across a straight cut, one of those edges must have the colors $d, \bar{d}$. We must rule out cases (i)-(iv). Consider first case (i). From Lemma 3 and the edges Source $\stackrel{2}{\rightarrow} Q \stackrel{1}{\rightarrow} R \stackrel{2}{\rightarrow}$ Sink, we have $h($ Source $\stackrel{2}{\rightarrow} Q)=h(R \stackrel{2}{\rightarrow}$ Sink $)$. The straight cut $(V \backslash\{\operatorname{Sink}\},\{\operatorname{Sink}\})$ is crossed by the edges $R \stackrel{2}{\rightarrow}$ Sink and $c_{j} \stackrel{6}{\rightarrow}$ Sink, hence those edges must have colors that are all different. We conclude $h($ Source $\stackrel{2}{\rightarrow} Q) \nsubseteq \not h\left(c_{j} \stackrel{6}{\rightarrow}\right.$ Sink $)$. Consider then case (ii). The straight cut $(V \backslash\{\operatorname{Sink}\},\{$ Sink $\})$ is crossed by the edges $s_{i 0} \stackrel{1}{\rightarrow}$ Sink and $c_{j} \stackrel{6}{\rightarrow}$ Sink, hence those edges must have colors that are all different. From the edges Source $\stackrel{2}{\rightarrow}$ $s_{i 0} \stackrel{1}{\rightarrow}$ Sink and Lemma 3 we have that $h\left(s_{i 0} \stackrel{1}{\rightarrow}\right.$ Sink $) \subseteq h\left(\right.$ Source $\left.\stackrel{2}{\rightarrow} s_{i 0}\right)$. We conclude $h\left(\right.$ Source $\left.\stackrel{2}{\rightarrow} s_{i 0}\right) \nsubseteq h\left(c_{j} \stackrel{6}{\rightarrow}\right.$ Sink $)$. Consider then cases (iii)-(iv): the proofs are similar to the proof of (ii), we omit the details. 
From Claim 1 we have two cases. We will consider each case in turn.

First suppose there exists $i, y$ such that $h\left(\right.$ Source $\left.\stackrel{2}{\rightarrow} s_{i y}\right) \subseteq h\left(c_{j} \stackrel{6}{\rightarrow}\right.$ Sink $)$. We begin by proving three properties of $h$ :

- Claim 2: $x_{i}$ appears in $c_{j}$.

- Claim 3: $h($ Source $\stackrel{2}{\rightarrow} Q)=h(Q \stackrel{1}{\rightarrow} R) \cup h\left(b_{i} \stackrel{1}{\rightarrow} a_{i+1}\right)$.

- Claim 4: $h\left(x_{i y} \stackrel{1}{\rightarrow} x_{i(y+1)}\right) \subseteq h\left(\right.$ Source $\left.\stackrel{2}{\rightarrow} s_{i y}\right)$.

Proof of Claim 2. The $k^{t h}$ occurrence of $x_{i}$ is in $c_{r}$ for some $r$, and $\operatorname{sg}(F)$ contains the edges $s_{i y} \stackrel{1}{\rightarrow} c_{r}$ and $x_{i(y+1)} \stackrel{1}{\rightarrow} c_{r}$. We must show $j=r$. Suppose $j \neq r$. The straight cut $\left(V \backslash\left\{c_{r}, \operatorname{Sink}\right\},\left\{c_{r}, \operatorname{Sink}\right\}\right)$ is crossed by the edges $c_{j} \stackrel{6}{\rightarrow}$ Sink and $s_{i y} \stackrel{1}{\rightarrow} c_{r}$, hence those edges must have colors that are all different. However, from Lemma 3 and $h\left(\right.$ Source $\left.\stackrel{2}{\rightarrow} s_{i y}\right) \subseteq h\left(c_{j} \stackrel{6}{\rightarrow}\right.$ Sink $)$, we have that $h\left(s_{i y} \stackrel{1}{\rightarrow} c_{r}\right) \subseteq h\left(\right.$ Source $\left.\stackrel{2}{\rightarrow} s_{i y}\right) \subseteq h\left(c_{j} \stackrel{6}{\rightarrow}\right.$ Sink $)$, a contradiction. We conclude $j=r$.

Proof of Claim 3. From Lemma $3, h($ Source $\stackrel{2}{\rightarrow} Q)=h(Q \stackrel{1}{\rightarrow} R) \cup h\left(Q \stackrel{1}{\rightarrow} a_{1}\right)$. The color in $h\left(Q \stackrel{1}{\rightarrow} a_{1}\right)$ takes a path from $Q$ to $R$ that contains the edge $b_{i} \stackrel{1}{\rightarrow} a_{i+1}$. From Lemma 3 we have that $h\left(Q \stackrel{1}{\rightarrow} a_{1}\right)=h\left(b_{i} \stackrel{1}{\rightarrow} a_{i+1}\right)$. From $h($ Source $\stackrel{2}{\rightarrow} Q)=h(Q \stackrel{1}{\rightarrow} R) \cup h\left(Q \stackrel{1}{\rightarrow} a_{1}\right)$ and $h\left(Q \stackrel{1}{\rightarrow} a_{1}\right)=h\left(b_{i} \stackrel{\vec{m}}{\rightarrow} a_{i+1}\right)$ we have $h($ Source $\stackrel{2}{\rightarrow} Q)=h(Q \stackrel{1}{\rightarrow} R) \cup h\left(b_{i} \stackrel{1}{\rightarrow} a_{i+1}\right)$.

Proof of Claim 4. From Claim 2 we have that $x_{i}$ appears in $c_{j}$. From Lemma 3 we have that we can find a color $d$ such that $h\left(\right.$ Source $\left.\stackrel{2}{\rightarrow} s_{i y}\right)=\{d, \bar{d}\}$ and $h\left(s_{i y} \stackrel{1}{\rightarrow} c_{j}\right)=\{d\}$. We have that the literals in clause $c_{j}$ are distinct so when we consider the edges $x_{i y} \stackrel{1}{\rightarrow} c_{r}$ and $x_{i(y+1)} \stackrel{1}{\rightarrow} c_{j}$, we have that $r \neq j$. From $h\left(\right.$ Source $\left.\stackrel{2}{\rightarrow} s_{i y}\right) \subseteq h\left(c_{j} \stackrel{6}{\rightarrow}\right.$ Sink $)$ we have that $\bar{d}$ is a color of some edge to $c_{j}$ and hence not a color of any edge to $c_{r}$. The vertex $x_{i y}$ has two outgoing edges $x_{i y} \stackrel{1}{\rightarrow} x_{i(y+1)}$ and $x_{i y} \stackrel{1}{\rightarrow} c_{r}$, so $h\left(x_{i y} \stackrel{1}{\rightarrow} x_{i(y+1)}\right)=\{\bar{d}\}$. So we have $h\left(x_{i y} \stackrel{1}{\rightarrow} x_{i(y+1)}\right) \subseteq h\left(\right.$ Source $\left.\stackrel{2}{\rightarrow} s_{i y}\right)$.

Finally, we will show that $\psi$ satisfies $c_{j}$. From Claim 4 we have $h\left(x_{i y} \stackrel{1}{\rightarrow}\right.$ $\left.x_{i(y+1)}\right) \subseteq h\left(\right.$ Source $\left.\stackrel{2}{\rightarrow} s_{i y}\right)$. The edges Source $\stackrel{2}{\rightarrow} Q$ and Source $\stackrel{2}{\rightarrow} s_{i y}$ both cross the straight cut $(\{$ Source $\}, V \backslash\{$ Source $\})$ and hence have colors that are all different. We thus have that $h\left(x_{i y} \stackrel{1}{\rightarrow} x_{i(y+1)}\right) \not \subset h($ Source $\stackrel{2}{\rightarrow} Q)$. From $h\left(x_{i y} \stackrel{1}{\rightarrow} x_{i(y+1)}\right) \nsubseteq h($ Source $\stackrel{2}{\rightarrow} Q)$ and Claim 3 we have that $h\left(x_{i y} \stackrel{1}{\rightarrow} x_{i(y+1)}\right) \neq$ $h\left(b_{i} \stackrel{1}{\rightarrow} a_{i+1}\right)$. Notice that $b_{i}$ has just two incoming edges, and that from $a_{i}$ to $b_{i}$ we have two paths: one path consists of vertices $a_{i}, x_{i 1}, \ldots, x_{i\left(p_{i}+1\right)}, b_{i}$; the other of vertices $a_{i}, \bar{x}_{i 1}, \ldots, \bar{x}_{i\left(q_{i}+1\right)}, b_{i}$. From Lemma 3 we have that the color of $h\left(b_{i} \stackrel{1}{\rightarrow} a_{i+1}\right)$ must be assigned to all edges on exactly one of these paths. We have already established $h\left(x_{i y} \stackrel{1}{\rightarrow} x_{i(y+1)}\right) \neq h\left(b_{i} \stackrel{1}{\rightarrow} a_{i+1}\right)$, so $h\left(a_{i} \stackrel{1}{\rightarrow} \bar{x}_{i 1}\right)=$ $h\left(b_{i} \stackrel{1}{\rightarrow} a_{i+1}\right)$. From $h\left(a_{i} \stackrel{1}{\rightarrow} \bar{x}_{i 1}\right)=h\left(b_{i} \stackrel{1}{\rightarrow} a_{i+1}\right)$ and Claim 3 we conclude $\psi\left(x_{i}\right)=$ true. From Claim 2 we have that $x_{i}$ appears in $c_{j}$. From $\psi\left(x_{i}\right)=$ true and that $x_{i}$ appears in $c_{j}$, we conclude that $\psi$ satisfies $c_{j}$.

Second suppose there exists $i, h$ such that $h\left(\right.$ Source $\left.\stackrel{2}{\rightarrow} \bar{s}_{i h}\right) \subseteq h\left(c_{j} \stackrel{6}{\rightarrow} \operatorname{Sink}\right)$. The proof is similar to the proof of the first case, we omit the details. 


\section{Main result}

Theorem 1. For straight-line programs, the core aliased register allocation problem with alignment restrictions is NP-complete.

Proof. First, the problem is in NP because a register assignment can be verified in polynomial time. Second, the problem is NP-hard because Lemmas 5, 4, and 1 give a chain of reductions 3 -SAT $\leq_{P}$ ACFLOW $\leq_{P}$ A12WIG $\leq_{P}$ CARAAR.

Corollary 1. ACFLOW and A12WIG are NP-complete.

\section{A Proof of Stockmeyer's Theorem}

Stockmeyer proved a result closely related to ours, namely that the shipbuilding problem is NP-complete [10, Application 9.1, p.204]. The shipbuilding problem for intervals with weights one or two is:

1-2-COLORING OF WEIGHTED INTERVAL GRAPHS (12WIG):

Instance: a weighted interval graph $G$ and a number $2 K$.

Question: does $G$ have a 1-2-coloring with $2 K$ colors?

Stockmeyer proved that 12 WIG is NP-complete [10, Remark 1, p.204]. As far as we are aware, our proof below (Theorem 2 ) is the first publicly available proof of Stockmeyer's theorem.

Figure 2(a) shows a graph that has a 1-2-coloring with 4 colors but no aligned 1-2-coloring with 4 colors. Hence, we have a case of a graph and a number of colors for which A12WIG is unsolvable while $12 \mathrm{WIG}$ is solvable.

We will reduce A12WIg to 12 WIG. From an instance $(G, 2 K)$ of A12WIG, we define a weighted interval graph uag $(G, 2 K)$ in the following way. Let $s$ be the minimum of the start points of all intervals in $G$ and let $e$ be the maximum of the end points of all intervals in $G$. The graph uag $(G, 2 K)$ consists of all the intervals in $G$ and for each $i, 0 \leq i<K$, the following intervals:

$$
\begin{array}{rlrl}
\text { weight two: } \delta_{2 i} & = & {[e, e+2 K+i[} \\
\text { weight two: } \delta_{2 i+1} & = & {[s, e+3 K+i[} \\
\text { weight one: } \gamma_{4 i} & =[e+2 K+i, e+5 K[ \\
\text { weight one: } \gamma_{4 i+1} & =[e+2 K+i, e+4 K+i[ \\
\text { weight one: } \gamma_{4 i+2} & =[e+3 K+i, e+4 K+i[ \\
\text { weight one: } \gamma_{4 i+3} & =[e+3 K+i, e+5 K[ \\
\text { weight two: } \quad \beta_{i} & =[e+4 K+i, e+5 K[
\end{array}
$$

Notice that for all $p, e \leq p<e+5 K$, the total weight of the intervals that contain $p$ is $4 K$.

Lemma 6. A $12 \mathrm{WIG} \leq_{P} 12 \mathrm{WIG}$. 


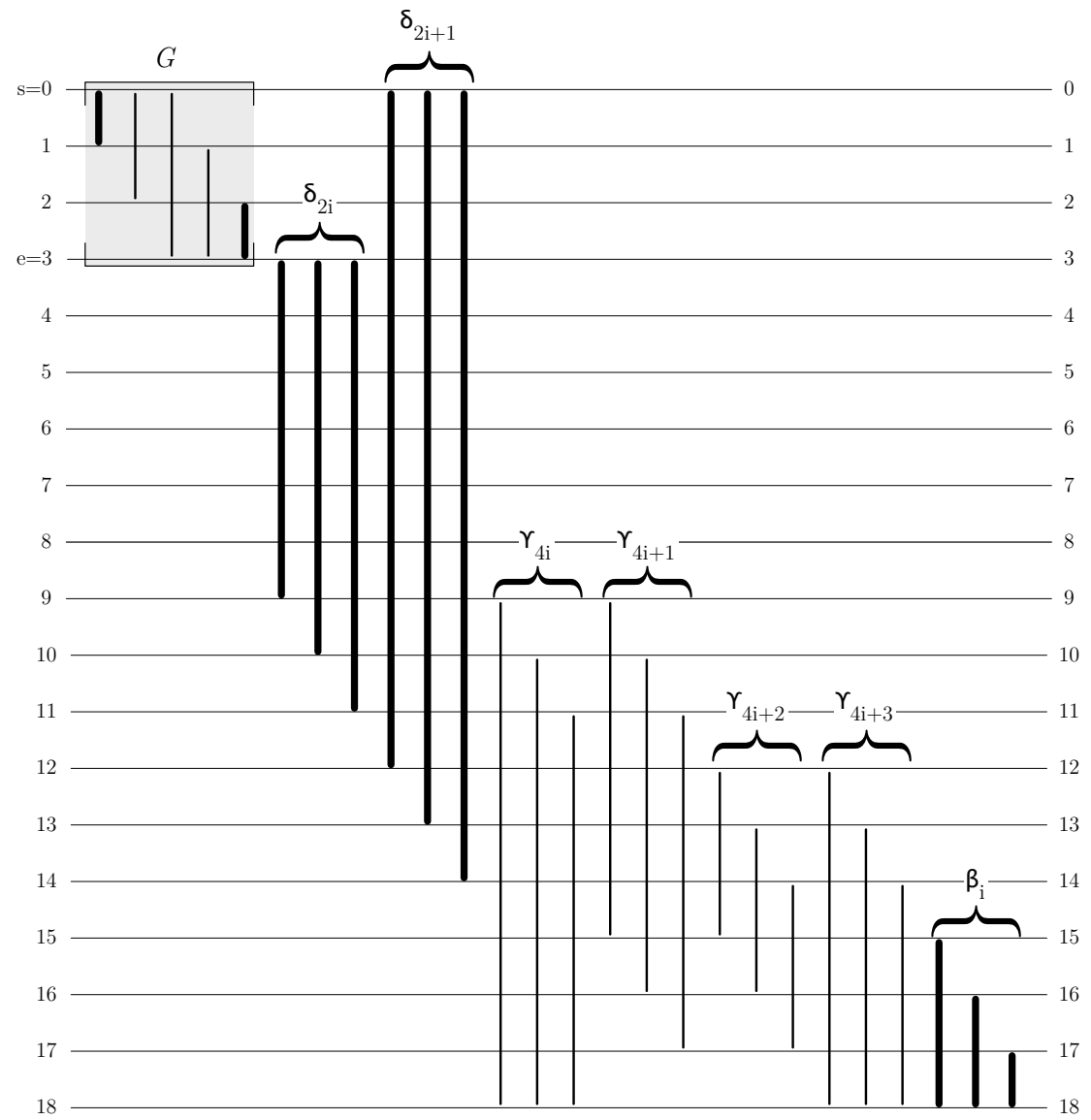

Fig. 7. From $G$ to uag $(G, 2 K)$. $K=3$. Intervals of weight two are bold.

Proof. Let $H$ denote the weighted interval graph with no intervals. From an instance $(G, 2 K)$ of A12wIG where $G$ has $q$ intervals, if $2 K>q$, then we construct the instance $(H, 1)$ of $12 \mathrm{WIG}$, otherwise, if $2 K \leq q$, we construct the instance (uag $(G, 2 K), 4 K)$ of 12 wig. The graph (uag $(G, 2 K)$ has $q+7 K \leq q+3.5 q=4.5 q$ intervals and we can construct both $(H, 1)$ and $(\operatorname{uag}(G, 2 K), 4 K)$ in polynomial time.

We now have two cases. In the case of $2 K>q$, we have that both $(G, 2 K)$ and $(H, 1)$ are solvable.

In the case of $2 K \leq q$, we will prove that $G$ has an aligned 1-2-coloring with $2 K$ colors if and only if uag $(G, 2 K)$ has a 1-2-coloring with $4 K$ colors.

$\Rightarrow$ ) Let $\kappa$ be an aligned 1-2-coloring of $G$ with $2 K$ colors. We define a function $m$ as follows. For $0 \leq i<K$, let $m(\{2 i\})=\{4 i\}$, let $m(\{2 i+1\})=\{4 i+1\}$, 
and let $m(\{2 i, 2 i+1\})=\{4 i, 4 i+1\}$. We define a mapping $\theta$ from vertices of $\operatorname{uag}(G, 2 K)$ to sets of colors:

$$
\begin{aligned}
\theta(\alpha) & =m(\kappa(\alpha)), \text { for each } \alpha \text { in } G \\
\theta\left(\delta_{2 i}\right) & =\{4 i, 4 i+1\} \\
\theta\left(\delta_{2 i+1}\right) & =\{4 i+2,4 i+3\} \\
\theta\left(\gamma_{4 i}\right) & =\{4 i\} \\
\theta\left(\gamma_{4 i+1}\right) & =\{4 i+1\} \\
\theta\left(\gamma_{4 i+2}\right) & =\{4 i+2\} \\
\theta\left(\gamma_{4 i+3}\right) & =\{4 i+3\} \\
\theta\left(\beta_{i}\right) & =\{4 i+1,4 i+2\}
\end{aligned}
$$

It is straightforward to check that $\theta$ is a 1-2-coloring of $\operatorname{uag}(G, 2 K)$ with $4 K$ colors.

$\Leftarrow)$ Let $\theta$ be a 1-2-coloring of uag $(G, 2 K)$ with $4 K$ colors. We first prove five properties of $\theta$ :

- Claim 1: For each $j, 0 \leq j<2 K, \theta\left(\delta_{j}\right)$ is a set of two aligned colors.

- Claim 2: For each $i, 0 \leq i<K$ :

- (a) $\theta\left(\delta_{2 i}\right)=\theta\left(\gamma_{4 i}\right) \cup \theta\left(\gamma_{4 i+1}\right)$

- (b) $\theta\left(\beta_{i}\right)=\theta\left(\gamma_{4 i+1}\right) \cup \theta\left(\gamma_{4 i+2}\right)$.

- (c) $\theta\left(\delta_{2 i+1}\right)=\theta\left(\gamma_{4 i+2}\right) \cup \theta\left(\gamma_{4 i+3}\right)$

- Claim 3: For each $i, 0 \leq i<K$, there exists $k, 0 \leq k<K$, such that:

$$
\begin{aligned}
\theta\left(\gamma_{4 i}\right) & =\{4 k\} \\
\theta\left(\gamma_{4 i+1}\right) & =\{4 k+1\} \\
\theta\left(\gamma_{4 i+2}\right) & =\{4 k+2\} \\
\theta\left(\gamma_{4 i+3}\right) & =\{4 k+3\}
\end{aligned}
$$

- Claim 4: $\cup_{i=0}^{k-1} \theta\left(\delta_{2 i+1}\right)=\{4 k+2,4 k+3 \mid 0 \leq k<K\}$.

- Claim 5: $\cup_{\alpha \in G} \theta(\alpha) \subseteq\{4 k, 4 k+1 \mid 0 \leq k<K\}$.

Proof of Claim 1 . The $2 K$ intervals $\delta_{j}, 0 \leq j<2 K$, all overlap. Given that $\theta$ has just $4 K$ colors available, the only way it can assign colors to those intervals is to assign each of them a set of aligned colors.

Proof of Claim 2. (a) For each $i, 0 \leq i<K$, the interval $\delta_{2 i}$ meets the intervals $\gamma_{4 i}$ and $\gamma_{4 i+1}$ in the point $e+2 K+i$, and no other intervals start or end at $e+2 K+i$. Given that the total weight of the intervals that contain $e+2 K+i$ is $4 K$, we have the claimed equation. We can prove (b) and (c) in a manner similar to the proof of (a); we omit the details.

Proof of Claim 3. From Claims $1+2$ we have that for every $i, 0 \leq i<K, \theta$ assigns a pair of aligned colors to $\gamma_{4 i}$ and $\gamma_{4 i+1}, \theta$ assigns a pair of consecutive colors to $\gamma_{4 i+1}$ and $\gamma_{4 i+2}$, and $\theta$ assigns a pair of aligned colors to $\gamma_{4 i+2}$ and $\gamma_{4 i+3}$. From those observations, we have the claimed equations.

Proof of Claim 4. We have that all the intervals $\delta_{2 i+1}$ overlap, for $0 \leq i<K$, so $\theta$ assigns them disjoint sets of colors. From that observation, Claim 2(c), and Claim 3 we have the claimed equation. 
Proof of Claim 5. Each interval $\alpha \in G$ overlaps with all the intervals $\delta_{2 i+1}$ so from Claim 4 we have that only colors of the form $4 k$ and $4 k+1$, for $0 \leq k<K$, are available to $\theta$ to assign to $\alpha$.

Let $m^{-1}$ be the inverse of the function $m$ defined above. Let $\kappa=m^{-1} \circ \theta$, restricted to $G$. From the definition of $\kappa$ and Claim 5 we have that the range of $\kappa$ is $\{j \mid 0 \leq j<2 K\}$ and that $\kappa$ must assign every interval of weight two in $G$ an aligned pair of colors. We conclude that $\kappa$ is an aligned 1-2-coloring of $G$ with $2 K$ colors.

Theorem 2. 1-2-coloring of weighted interval graphs is NP-complete.

Proof. First, the problem is in NP because a 1-2-coloring can be verified in polynomial time. Second, the problem is NP-hard because Lemmas 5, 4, and 6 give a chain of reductions $3-\mathrm{SAT} \leq_{P}$ ACFLOW $\leq_{P}$ A12 WIG $\leq_{P} 12 \mathrm{wIG}$.

\section{Conclusion}

We have shown that aliased register allocation with alignment restrictions is difficult, even for straight-line programs where each variable has at most one definition point. Our result confirms the need for the heuristics and worst-case exponential time methods for aliased register allocation that are used today.

In this paper we have considered register allocation as a decision problem. We can also view register allocation as an optimization problem: minimize the number of registers. Open problem: give nontrivial upper and lower bounds on the approximability of our register allocation problem. For example, is our register allocation problem APX-hard?

Acknowledgment. A preliminary version of this paper was presented at ICALP 2007. We thank the TCS reviewers for helpful suggestions. We were supported in part by a National Science Foundation Award number 0401691. Fernando Pereira was sponsored by the Brazilian Ministry of Education under grant number 218603-9.

\section{Appendix: Illustrations}

In this section we illustrate the edges in $\operatorname{sg}(F)$. The illustrations show the edges and colors to and from each vertex except Source and Sink. For vertices where the colors depend on the value of $\psi\left(x_{i}\right)$, we use the same figure to show both the case when $\psi\left(x_{i}\right)$ is true and the case when $\psi\left(x_{i}\right)$ is false. 

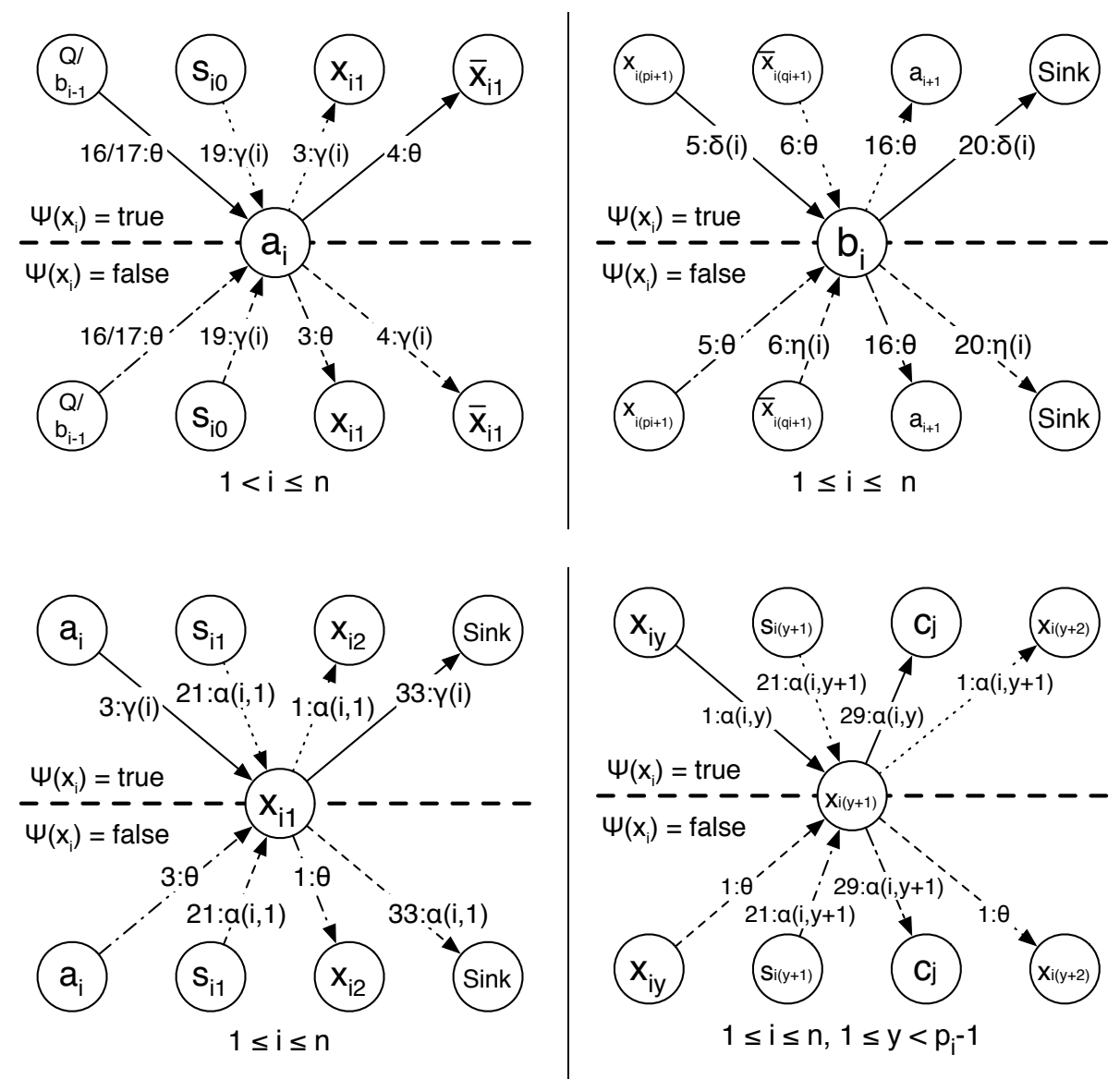

Fig. 8. Four illustrations of edges in $\operatorname{sg}(F)$. 

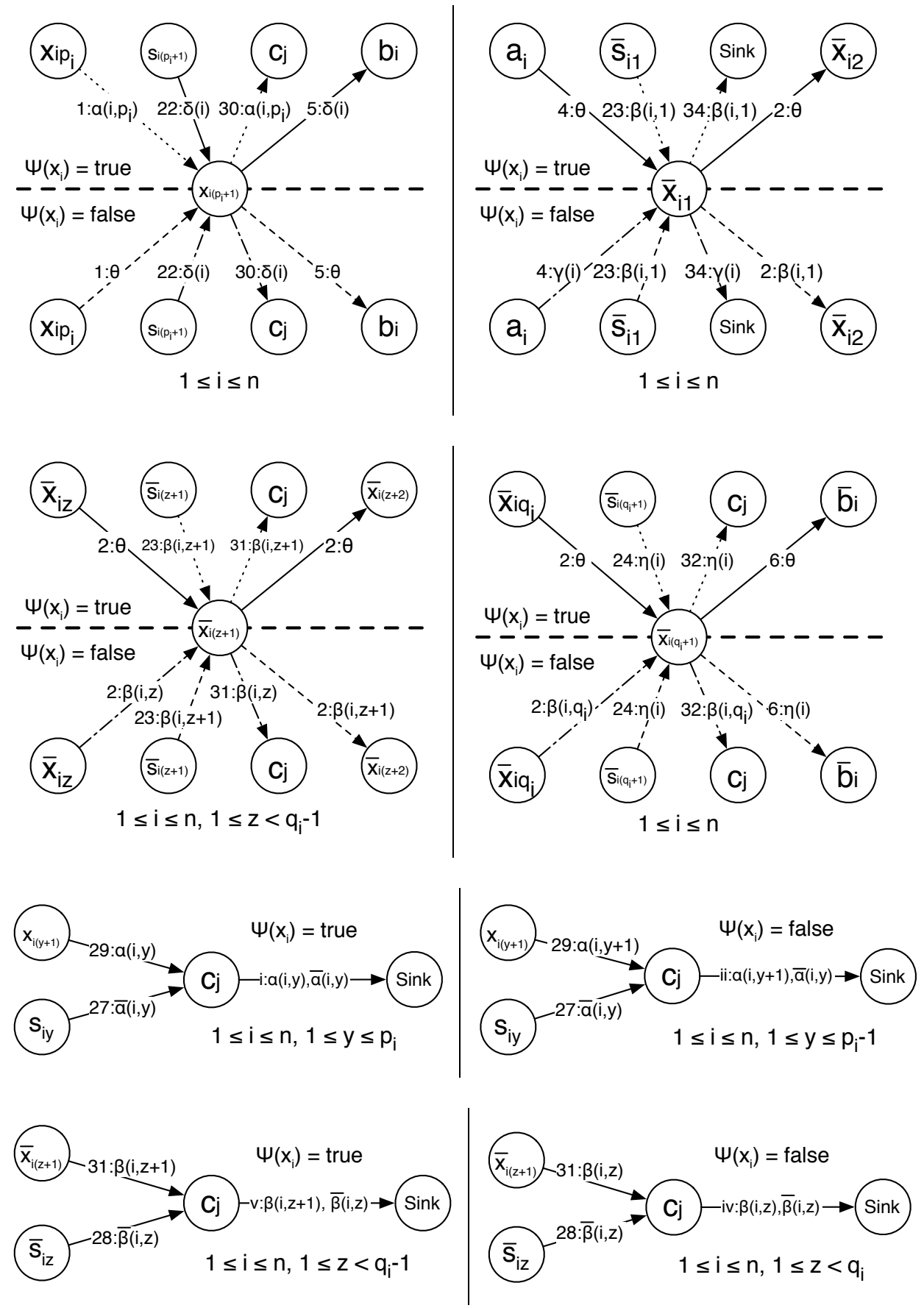

Fig. 9. Eight illustrations of edges in $\operatorname{sg}(F)$. 

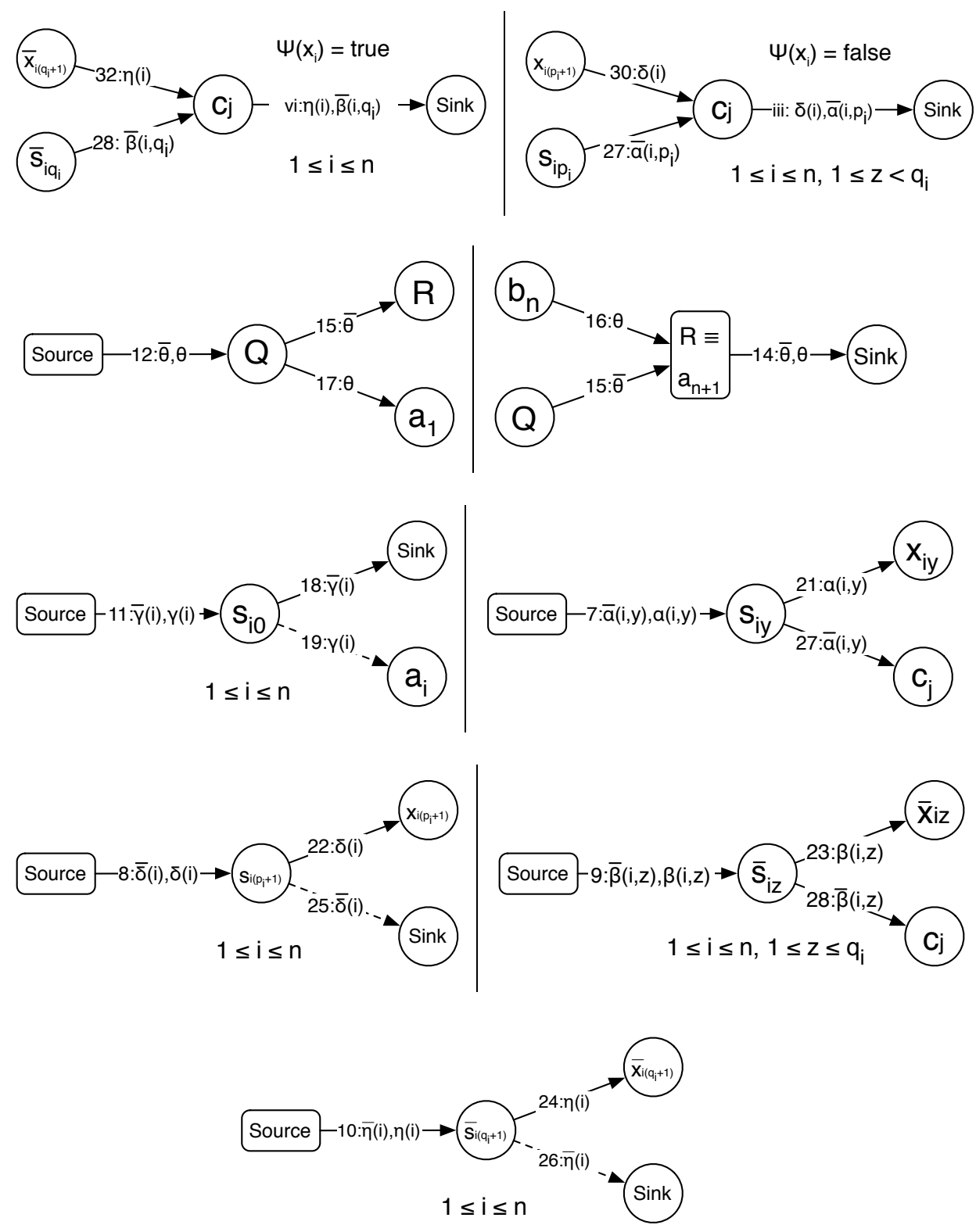

Fig. 10. Nine illustrations of edges in $\operatorname{sg}(F)$. 


\section{References}

1. M. Biró, M. Hujter, and Zs. Tuza. Precoloring extension. I:interval graphs. In Discrete Mathematics, pages 267-279. ACM Press, 1992. Special volume (part 1) to mark the centennial of Julius Petersen's "Die theorie der regularen graphs".

2. Preston Briggs. Register Allocation via Graph Coloring. PhD thesis, Rice University, 1992.

3. Preston Briggs, Keith Cooper, and Linda Torczon. Coloring register pairs. ACM Letters on Programming Languages, 1(1):3-13, 1992.

4. Gregory J. Chaitin, Mark A. Auslander, Ashok K. Chandra, John Cocke, Martin E. Hopkins, and Peter W. Markstein. Register allocation via coloring. Computer Languages, 6:47-57, 1981.

5. Lin Chen. Optimal parallel time bounds for the maximum clique problem on intervals. Information Processing Letters, 42(4):197-201, 1992.

6. Thomas H. Cormen, Charles E. Leiserson, Ronald L. Rivest, and Clifford Stein. Introduction to Algorithmics. McGraw-Hill, 2001. Second edition.

7. S. Even, A. Itai, and A. Shamir. On the complexity of timetable and multicommodity flow problems. SIAM Journal on Computing, 5(4):691-703, December 1976.

8. Martin Farach and Vincenzo Liberatore. On local register allocation. In 9th ACMSIAM Symposium on Discrete Algorithms, pages 564-573. ACM Press, 1998.

9. Christopher Fraser and David Hanson. A Retargetable C Compiler: Design and Implementation. Addison-Wesley, 1995.

10. Martin Charles Golumbic. Algorithmic Graph Theory and Perfect Graphs. Elsevier, 2nd edition, 2004.

11. Ulrich Hirnschrott, Andreas Krall, and Bernhard Scholz. Graph coloring vs. optimal register allocation for optimizing compilers. In JMLC, Modular Programming Languages, Joint Modular Languages Conference, pages 202-213. Springer, 2003.

12. Timothy Kong and Kent D. Wilken. Precise register allocation for irregular architectures. In Internation Symposium on Microarchitecture, pages 297-307. ACM, 1998.

13. Akira Koseki, Hideaki Komatsu, and Toshio Nakatani. Preference-directed graph coloring. In PLDI, ACM SIGPLAN Conference on Programming Language Design and Implementation, pages 297-307. ACM, 2002.

14. V. Krishna Nandivada, Fernando Magno Quintão Pereira, and Jens Palsberg. A framework for end-to-end verification and evaluation of register allocators. In Proceedings of SAS'0\%, International Static Analysis Symposium, pages 153-169, Kongens Lyngby, Denmark, August 2007.

15. Brian R. Nickerson. Graph coloring register allocation for processors with multiregister operands. In PLDI, ACM SIGPLAN Conference on Programming Language Design and Implementation, pages 40-52, 1990.

16. Johan Runeson and Sven-Olof Nystrom. Retargetable graph-coloring register allocation for irregular architectures. In SCOPES, Software and Compilers for Embedded Systems, pages 240-254. Springer, 2003.

17. Anita Saha, Madhumangal Pal, and Tapan K. Pal. Selection of programme slots of television channels for giving advertisement: A graph theoretic approach. Information Sciences, 177(12):2480-2492, 2007.

18. Bernhard Scholz and Erik Eckstein. Register allocation for irregular architectures. In LCTES/SCOPES, Joint Conference on Languages, Compilers, and Tools for Embedded Systems and Software and Compilers for Embedded Systems, pages 139148. ACM, 2002. 
19. Michael D. Smith, Norman Ramsey, and Glenn Holloway. A generalized algorithm for graph-coloring register allocation. In PLDI, ACM SIGPLAN Conference on Programming Language Design and Implementation, pages 277-288, 2004.

20. Andrew W. Appel with Jens Palsberg. Modern Compiler Implementation in Java. Cambridge University Press, 2002. 